\title{
Sparse conjugate directions pursuit with application to fixed-size kernel models
}

\author{
Peter Karsmakers • Kristiaan Pelckmans • \\ Kris De Brabanter • Hugo Van hamme • \\ Johan A.K. Suykens
}

Received: 27 February 2010 / Accepted: 16 May 2011 / Published online: 4 June 2011

(C) The Author(s) 2011

\begin{abstract}
This work studies an optimization scheme for computing sparse approximate solutions of over-determined linear systems. Sparse Conjugate Directions Pursuit (SCDP) aims to construct a solution using only a small number of nonzero (i.e. nonsparse) coefficients. Motivations of this work can be found in a setting of machine learning where sparse models typically exhibit better generalization performance, lead to fast evaluations, and might be exploited to define scalable algorithms. The main idea is to build up iteratively a conjugate set of vectors of increasing cardinality, in each iteration solving a small linear subsystem. By exploiting the structure of this conjugate basis, an algorithm is found (i) converging in at most $D$ iterations for $D$-dimensional systems, (ii) with computational complexity close to the classical conjugate gradient algorithm, and (iii) which is especially efficient when a few iterations suffice to produce a good approximation. As an example, the application of SCDP to Fixed-Size Least Squares Support Vector Machines (FS-LSSVM) is discussed resulting in a scheme which efficiently finds a good model size for the FSLSSVM setting, and is scalable to large-scale machine learning tasks. The algorithm is
\end{abstract}

Editors: Sūreyya Ōzōğūr-Akyūz, Devrim Unay, and Alex Smola.

P. Karsmakers $(\bowtie) \cdot$ K. De Brabanter $\cdot$ H. Van hamme · J.A.K. Suykens

Department of Electrical Engineering, K.U.Leuven, Kasteelpark Arenberg 10, 3001 Heverlee, Belgium e-mail: peter.karsmakers@esat.kuleuven.be

K. De Brabanter

e-mail: kris.debrabanter@esat.kuleuven.be

H. Van hamme

e-mail: hugo.vanhamme@esat.kuleuven.be

J.A.K. Suykens

e-mail: johan.suykens@esat.kuleuven.be

K. Pelckmans

Division of Systems and Control, Department of Information Technology, Uppsala University, 75105

Uppsala, Sweden

e-mail: kp@it.uu.se

P. Karsmakers

Department IBW, K.H.Kempen (Association K.U.Leuven), 2440 Geel, Belgium 
empirically verified in a classification context. Further discussion includes algorithmic issues such as component selection criteria, computational analysis, influence of additional hyper-parameters, and determination of a suitable stopping criterion.

Keywords Sparse approximation · Greedy heuristic · Matching pursuit · Linear system · Kernel methods

\section{Introduction}

Consider a task of determining a vector of parameters $w \in \mathbb{R}^{D}$ such that

$$
X w=y,
$$

where $y \in \mathbb{R}^{N}$, and the matrix $X \in \mathbb{R}^{N \times D}$ which typically contains $N$ measurements of size $D$. As illustrated in Fig. 1, three problem cases can be distinguished:

- Uniquely-determined linear system $(N=D)$ : When $X$ is of full rank and $N=D$, a single unique solution exists.

- Over-determined linear system $(N>D)$ : When $X$ is of full rank and $D<N$, no solution exist.

- Under-determined linear system $(D>N)$ : When $X$ is of full rank and $D>N$, infinitely many solutions exist.

The case that $N$ equals $D$ is not treated in this work. The other cases in general either have no or infinitely many solutions. This gives the opportunity to choose one that satisfies an appropriate criterion. Although this work focuses on over-determined systems, first an example of under-determined systems originating from the signal processing community is given.

In signal processing only recently a new area of sparse modeling of signals and images emerged (Bruckstein et al. 2009). Consider a data compression example. Here, one tries to transform raw data vectors to be represented in a new coordinate system. The reason why people are interested in this change of coordinate system is a potential sparse representation (i.e. the solution is expressed as a linear combination of the features using only a few nonzero coefficients) of the original inputs. In case of data compression the goal is to find the sparsest representation (which might be stored only using a small number of bits) given the new coordinate basis. This typically leads to under-determined systems which have infinitely many solutions. To narrow down the choice, one can include the requirement to additionally minimize the non-sparsity using the $L_{0}$-norm (which counts the number of nonzero elements). This gives

$$
w^{0, *}=\underset{w}{\arg \min }\|w\|_{0} \quad \text { such that } \quad X w=y,
$$

where $X \in \mathbb{R}^{N \times D}$ with $N \ll D$ whose columns are the elements of the different bases to be used in the representation, $y$ the vector of signal values to be represented, and $w$ the coefficients in the coordinate system defined by $X$ to represent $y$.

Often the exact constraint $X w=y$ is relaxed to tolerate a slight discrepancy between $X w$ and $y$. In case the $L_{2}$ norm is used for evaluating the error, the approximation of the optimization problem in the under-determined setting becomes

$$
w^{0, *}=\underset{w}{\arg \min }\|w\|_{0} \quad \text { such that } \quad\|X w-y\|_{2}^{2} \leq \delta .
$$




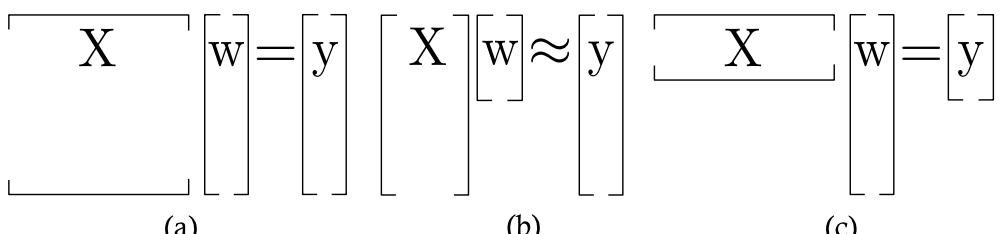

(a)

(b)

(c)

Fig. 1 Schematical representation of (a) an uniquely defined system of linear equations; (b) an over-determined system of linear equations; (c) an under-determined system of linear equations

This work focuses on solving over-determined systems originating from least squares estimation problems with sparse solutions. As will be explained next, this goal can be formulated such as given in (3). Since over-determined systems in general cannot be exactly solved, one typically prefers the solution solving the system (1) with minimal least squares residuals $r=X w-y$, or

$$
w^{*}=\underset{w}{\arg \min } \frac{1}{2}\|X w-y\|_{2}^{2},
$$

which is equivalent to solving the normal equations defined as

$$
A w=b,
$$

if $A$ is invertible and where we define $A=X^{T} X \in \mathbb{R}^{D \times D}$ and $b=X^{T} y \in \mathbb{R}^{D}$. Several methods have been developed for solving such sets of linear equations. See the reference works Press et al. (1993) and citations. One of the most successful iterative algorithm for solving such large linear systems is the Conjugate Gradient (CG) algorithm. The CG method for linear systems was first proposed by Hestenes and Stiefel (1952) in the 1950s as an iterative method for solving linear systems with positive definite matrices. Later on, the same ideas were used for solving non-convex optimization problems, notably in a context of artificial neural networks (Möller 1993).

Solving (4) will not result in a sparse solution. The goal of this work is to devise an algorithm which approximately solves (5) resulting in a sparse solution for $w$. Our motivations to obtain a sparse approximative solution to (4) are:

- When dealing with estimation problems, sparse coefficients can be interpreted typically as a form of feature selection.

- When working in the context of machine learning, sparse predictor rules lead typically to improved generalization (Floyd and Warmuth 1995; Vapnik 1998).

- Sparseness can often be exploited to yield more computation and memory-efficient numerical techniques, e.g. for evaluating the estimated model.

- Sparseness in the solution might be exploited when scaling algorithms up to handle large data sets.

In order to obtain a sparse solution for $w$ in (4), one may again search for the minimal $L_{0}$-norm of a solution approximatively solving (4). In that case, the objective reduces again to (3), and we might as such handle the over-determined case by similar tools as those used for the under-determined case. The aim of the present work is to devise a computationally efficient algorithm which gives a sparse approximate solution of such a (possibly largescale) system. For this purpose CG is adapted such that the iteratively constructed conjugate basis yields solutions of increasing cardinality. In case the algorithm can be terminated in 
less than $D$ steps a sparse solutions is obtained. The resulting algorithm will be called Sparse Conjugate Directions Pursuit (SCDP).

A second focus of the paper is to apply SCDP in the context of Least Squares Support Vector Machines (LS-SVMs) (Suykens et al. 2002b). Compared to Support Vector Machines (SVMs) (Vapnik 1998), LS-SVMs are based on solving a system of linear equations instead of solving a Quadratic Program (QP) problem. Although learning can be performed using a simpler optimization strategy, the LS-SVM solution is not sparse as opposed to SVM. In order to obtain faster model evaluation, in Suykens et al. (2000, 2002a) the authors proposed a pruning mechanism based on sorting the absolute values of the elements of the solution of LS-SVM. A more sophisticated pruning scheme is explained in de Kruif and de Vries (2003) for function estimation and in Zeng and Chen (2005) a Sequential Minimization Optimization (SMO) based pruning method is proposed. However, in case of large-scale problems these methods are not feasible since they all solve a large linear system which is iteratively shrunken.

The proposed algorithm iteratively constructs a model with increasing cardinality and, if stopped early (number of iterations smaller than the dimension of the training examples), provides a sparse solution. For this purpose the primal formulation of the LS-SVM problem is first approximated using the ideas described in Suykens et al. (2002b), De Brabanter et al. (2010) to formulate a method called Fixed-Size LS-SVM (FS-LSSVM). This gives an over-determined linear system which might be solved using SCDP. We will discuss the connections to other methods found in the literature such as Kernel Matching Pursuit (KMP) (Vincent and Bengio 2002), Sparse Greedy Gaussian Process (SGGP) (Smola and Bartlett 2001), Orthogonal Least Squares (OLS) regression (Chen et al. 2009), other sparse LS-SVM versions (Cawley and Talbot 2002; Jiao et al. 2007).

The main contributions of this work can be summarized as follows:

- An integration of the conjugate gradient algorithm with matching pursuit (Mallat 1999).

- The use of SCDP for estimating fixed-size kernel models.

- A detailed description of all components of the model selection process including algorithmic parameters, and Cross-Validation (CV). As a result of this process a "good" model size is automatically obtained.

- An empirical comparison with other methods in terms of training complexity, classification performance, and sparseness.

Different approaches to solve (3) found in the literature are discussed in Sect. 2. Then details about, and relations to, existing alternatives of SCDP are given in Sect. 3. The application of the resulting algorithm to the LS-SVM framework is given in Sect. 4. The final algorithm is validated on several publicly available data sets as reported in Sect. 5 and conclusions are drawn in Sect. 6.

\section{Heuristics to obtain a sparse approximation}

In this section, methods to tackle the optimization described in (3) are briefly summarized without making a distinction whether or not the method originates from an under-determined or over-determined setting.

Since the problem defined in (3) is NP-hard in general (Natarajan 1995), the most efficient known algorithms use heuristics to accomplish results. One such heuristic is backward Stepwise Regression (BSR) (Hastie et al. 2001). Starting from a model obtained using all components, BSR sequentially deletes components from the solution. However, since one 
of the goals of this work is to tackle large scale problems for which solving the full problem (as required for BSR) might be unfeasible, backward selection techniques are disregarded. Instead methods applicable to large scale data are considered. A good survey of such methods is given in Bruckstein et al. (2009). We now proceed by briefly summarizing the key methods, and their relations.

\subsection{Greedy heuristic}

A first approach is to handle the problem heuristically as defined in (3) by greedy, stepwise algorithms. A greedy strategy avoids an exhaustive search for solving (3) in favor of a series of locally optimal single-term updates. In general, the approximation error is reduced in each iteration as much as possible given the starting approximation and the constraint that only one term can be selected at a single iteration. This explains the name "greedy". Examples include:

- A simple version in this class is called forward stepwise regression (FSR) in the statistics community. This is equivalent to Matching Pursuit (MP) in the signal processing and wavelet community (Mallat 1999). A principal scheme is shown in Algorithm 1. MP (FSR) decomposes any $y$ (e.g. signal) into a linear expansion of components (columns of $X$ ) that are selected from $X$. Consider the normal equation in (5). The algorithm starts with an empty active set (which is a set of indices associated to selected components) and an approximate solution ${ }^{1}$ vector $w^{(1)}=0_{D}, 0_{D}=(0, \ldots, 0)^{T} \in \mathbb{R}^{D}$ and then iteratively increments the cardinality of $w^{(k)}$. Given a collection of possible components, the one having the largest absolute correlation with the residuals, ${ }^{2} c^{(k)}=A w^{(k)}-b=X^{T} r^{(k)}$, is selected, and denoted $\mathrm{as}^{3}\left(c^{(k)}\right)_{j}$. The corresponding element in the parameter vector $(w)_{j}$ is then updated such that $\left(c^{(k+1)}\right)_{j}=0$. After calculating $c^{(k+1)}$ the process is repeated. The algorithm is stopped when an appropriate stopping criterion is reached, possibly resulting in a sparse parameter vector $w^{(k)}$.

- Because in the previous mentioned greedy algorithms only a single element of $w^{(k)}$ is updated in each iteration, in case of MP (FSR) the set of components selected in each iteration $(k \geq 2)$ is suboptimal in least squares sense and so are the corresponding elements of $w^{(k)}$. This can be corrected in each step using back-fitting (i.e. solving the linear system using the components selected so far), which is known as Orthogonal Matching Pursuit (OMP) (Pati et al. 1993). This is slightly different from Orthogonal Least Squares (OLS) (Chen et al. 1989) which has a different component selection strategy. While being computationally more demanding per iteration, often more accurate results are obtained compared to MP.

- MP (FSR) is a greedy fitting technique that can be overly greedy, perhaps eliminating at the next step useful components that happen to be correlated with the already selected components. Forward Stagewise Regression (FStR) is a more cautious version of FSR, which follows the same algorithm except that it only takes a fraction (using a shrinkage factor) of the FSR step (i.e. the update rules in Algorithm 1 for $w$ and $c$ change to $\left(w^{(k+1)}\right)_{j}=\left(w^{(k)}\right)_{j}-s_{\epsilon} \beta$ and $c^{(k+1)}=c^{(k)}+s_{\epsilon} a_{j} \beta$ with $s_{\epsilon}$ the shrinkage factor). This results in smaller steps which may increase the computation time.

\footnotetext{
${ }^{1}$ To indicate a dependency on the iteration number $k$, variables are marked by a superscript $(k)$.

${ }^{2}$ In case we mention residuals we indicate the residues from a linear system which in this work can be $r=X w-y$ or $c=A w-b$. Which linear system is considered should be clear from the context.

${ }^{3}$ Notation $(x)_{j}$ selects the $j$-th element of vector $x$.
} 
- Since greedy strategies add only a single component at each iteration to the active set, they require at least as many iterations as there are nonzero elements in the solution vector $w$. A recently proposed promising variant of OMP is called stagewise OMP (Donoho et al. 2006) which selects more than one component at each iteration using a thresholding procedure. Such approach can result in reducing the final number of iterations, but requires slightly more work in each iteration.

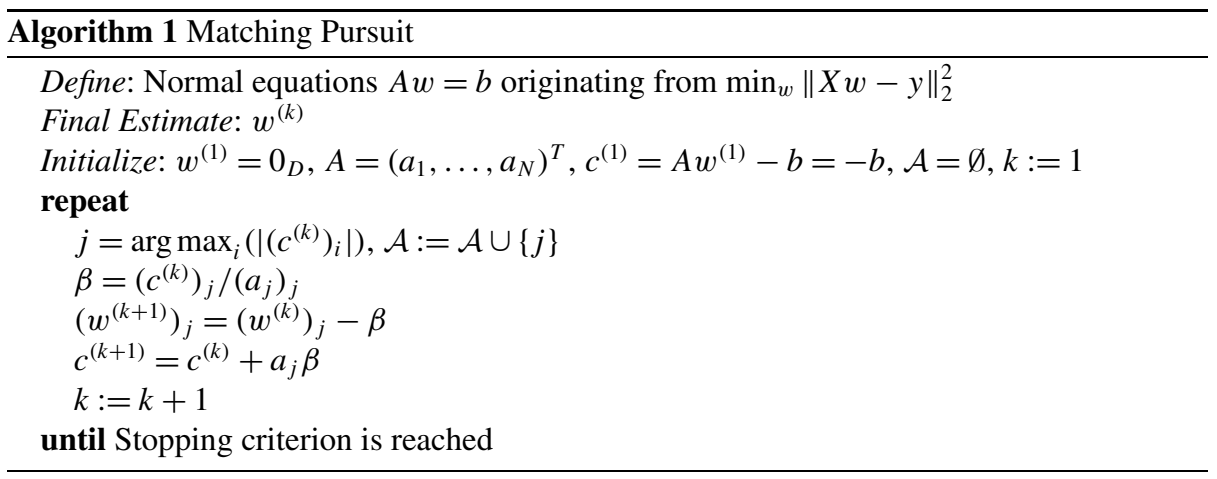

\section{$2.2 L_{1}$-norm heuristic}

Another approach is to relax the zero norm $\|\cdot\|_{0}$ to the convex $\|\cdot\|_{1}$, resulting in the class of $L_{1}$-regularized algorithms. Here, one solves the problem

$$
w^{*, 1}=\underset{w}{\arg \min }\|w\|_{1} \quad \text { such that } \quad\|X w-y\|_{2}^{2} \leq \delta,
$$

which is equivalent to the optimization problem

$$
\min _{w, t} \frac{1}{2}\|X w-y\|_{2}^{2}+\lambda\|w\|_{1}
$$

with $\lambda>0$. This type of relaxation is used in for instance the LASSO (Tibshirani 1996), ${ }^{4}$ for the purpose of model selection in statistics or Basis Pursuit (BP) (Chen et al. 1999) with applications such as compressed sensing (Candès 2006; Donoho 2006) and sparse signal recovery in signal processing (Donoho and Tsaig 2006). Because of the characteristics of these constraints, the formulation tends to produce coefficients which are exactly zero. Several special purpose solvers can be utilized to solve (7) such as

- Iteratively re-weighted Least Squares (IRLS) algorithms: by defining an appropriate Langrangian, (7) can be stated as an unconstrained minimization problem which can be solved by IRLS in case we write $\|w\|_{1} \equiv w^{T} W^{\dagger} w$ where $W=\operatorname{diag}(|w|)$, and $\dagger$ denotes a pseudoinverse (Daubechies et al. 2008). Given a current approximate solution of $w^{(k)}$, compute $W^{(k)}=\operatorname{diag}\left(\left|w^{(k)}\right|\right)$ and iteratively solve objective $\min _{w} 0.5\|X w-y\|_{2}^{2}+\lambda w^{T} W^{(k) \dagger} w$.

\footnotetext{
${ }^{4}$ LASSO was mainly developed in the over-determined case.
} 
- Iterative shrinkage methods: for large scale problems an approximate strategy is more realistic. Iterative shrinkage methods iteratively use a simple one dimensional operation that set small entries to zeros and shrinking the other entries towards zero. Assume a square unitary matrix $X$. As a first step find an intermediate solution $\tilde{w}=X^{T} y$, which hopefully has only a few large entries exceeding many small noisy ones. Secondly, apply shrinkage function $f\left((w)_{i} ; \lambda_{t}\right)=\operatorname{sign}\left((w)_{i}\right)\left(\left|(w)_{i}\right|-\lambda_{t}\right)_{+}{ }^{5}$ for $i=1, \ldots, D$. In the general case where $X$ is not unitary this idea is applied iteratively. These methods can compete with greedy methods both in simplicity and efficiency but are not yet as mature as the greedy alternatives see Bruckstein et al. (2009) and the reference therein for a discussion.

- Stepwise algorithms: are heuristic methods, inspired by $L_{1}$ solvers, which iteratively add or remove components from the active set. Examples are (Least Angle RegreSsion) LARS (Efron et al. 2004) and the homotopy method (Osborne et al. 2000). Consider the problem (7). It was observed in Efron et al. (2004), and Osborne et al. (2000) that the regularization path (i.e. the sequence of solutions, as $\lambda$ varies from 0 to $\infty$ ) is piecewise linear, changing only at critical values of $\lambda$. Each vertex on this regularization path indicates a different sparse solution, i.e. vectors having nonzero elements only on a subset of the potential components. At each vertex the active set is updated through the addition and removal of components. Based on this observation a heuristic algorithm was developed that, starting from an empty active set, iteratively adds and removes components. Note the difference with the greedy approaches which can only increase the number of indices in the active set. Once a component is in the active set it remains there.

- Dedicated solvers: by exploiting the structure of the resulting quadratic program, efficient dedicated solvers are described, see e.g. Kim et al. (2007) which uses a specialized interior-point algorithm, or Figueiredo et al. (2007) where the authors propose to use an efficient gradient projection algorithm.

Remark 1 Using an $L_{1}$ penalty introduces a bias which is often undesirable. As a consequence (i) the intermediate solutions are not exactly equal to the least squares estimates (ii) component selection is based on a biased estimator. To overcome the former, a de-bias step can be performed (Moghaddam et al. 2006; Figueiredo et al. 2007). After finding an approximate solution, the de-biased solution is found by fixing the sparsity pattern, and solving for the remaining coefficients using an ordinary least squares method. Put in other words, having selected the components, a reduced linear system is solved in these components alone. Greedy techniques are also biased in their component selection strategy but provide the intermediate least squares estimates directly.

\subsection{Relations}

In Efron et al. (2004) the LARS algorithm was proposed. LARS is a less greedy algorithm than OMP (FSR with back-fitting), it uses a similar strategy but only uses as much of a component as it "deserves":

- Consider the normal equations defined in (5) and let the index set of currently selected components be $\mathcal{A}=\left\{s_{1}, \ldots, s_{N_{A}}\right\}$, the set of not yet selected components $\overline{\mathcal{A}}$, selection matrix $\Sigma_{\mathcal{A}}=\left(e_{s_{1}}, \ldots, e_{s_{N_{A}}}\right), \Sigma_{\mathcal{A}} \in \mathbb{R}^{D \times N_{A}}$ where $e_{i} \in \mathbb{R}^{D}$ the $i$-th unit vector of size $D$, and a submatrix $A_{\mathcal{A}}=\Sigma_{\mathcal{A}}^{T} A \Sigma_{\mathcal{A}}$ of $A$.

\footnotetext{
${ }^{5}$ Operator $(\cdot)+$ sets negative values to zero while positives remain unchanged.
} 


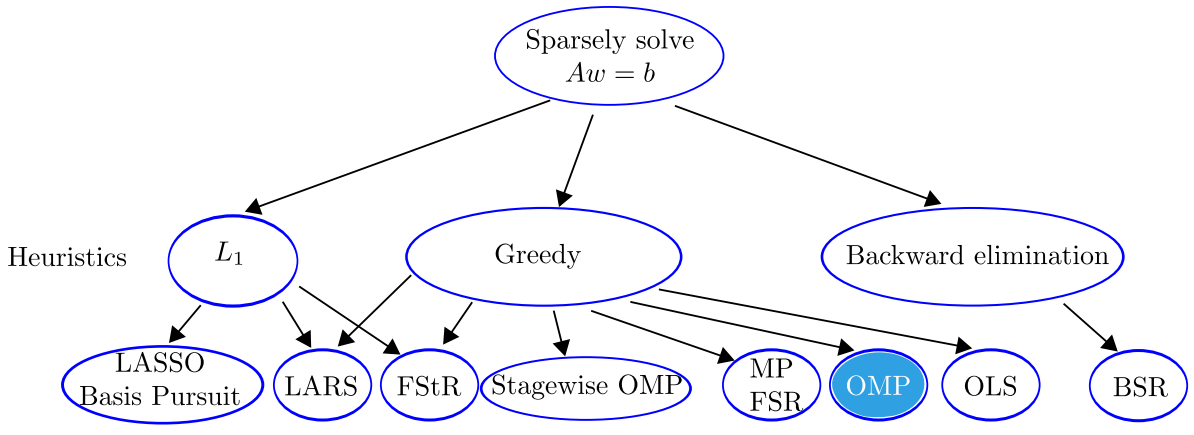

Fig. 2 Overview of different approaches to tackle (3)

- Start by selecting the $j^{*}$-th column of $X$ which correlates the most with $X w^{(1)}-y$ or $j^{*}=\arg \max _{j}\left|b_{j}\right|\left(\right.$ since $\left.w^{(1)}=0\right)$.

- The parameter vector $w^{(2)}$ is changed ${ }^{6}$ according to the direction defined by the joint least squares solution of the currently selected components $A_{\mathcal{A}} \Sigma_{\mathcal{A}}^{T} w^{(2)}-\Sigma_{\mathcal{A}}^{T} b=0$ until a competitor $j \in \overline{\mathcal{A}}$ has as much correlation with the current least squares residuals $\left(\left|A_{\mathcal{A}} \Sigma_{\mathcal{A}}^{T} w^{(2)}-\Sigma_{\mathcal{A}}^{T} b\right|\right)_{i}=\left|e_{j}^{T} A \Sigma_{\mathcal{A}} \Sigma_{\mathcal{A}}^{T} w^{(2)}-e_{j}^{T} b\right|$ for $i \in\left\{1, \ldots, N_{A}\right\}$.

- Then this new component is inserted in $\mathcal{A}$ and removed from $\overline{\mathcal{A}}$, and the process is continued.

The following observations bridged the gap between $L_{1}$-minimization and greedy OMP (Donoho and Tsaig 2006; Efron et al. 2004):

- Only a simple modification to LARS (when a nonzero coefficient hits zero, drop it from $\mathcal{A}$ and recompute the current joint least squares direction to LARS) is needed to make LARS exactly reproduce the LASSO regularization path (the resulting algorithm is very similar to the homotopy method described in Osborne et al. 2000).

- LARS and OMP are based on the same fundamental principle, which solves a sequence of least-squares problems on an increasingly larger subspace, defined by the active set.

This helps explaining the similarity of both approaches in several theoretical and numerical studies (Tropp 2004; Bruckstein et al. 2009). Since its simplicity and the fact that OMP has similar behavior as the more sophisticated $L_{1}$-norm based optimization methods, we now proceed by defining an efficient algorithm for (greedy) OMP and present in the experimental section that it can outperform an $L_{1}$ related alternative in terms of speed while having comparable accuracy. Figure 2 schematically summarizes the different approaches to tackle (3).

\section{Greedy heuristic: sparse conjugate directions}

This section describes a new approach to solve (3) which belongs to the category of greedy heuristics.

\footnotetext{
${ }^{6}$ In Efron et al. (2004) the authors observed that both LASSO and FStR behaved very similar. In an attempt to reduce the number of iterations of FStR, the authors defined LARS which "optimizes" the small FStR steps to larger steps, but smaller than the FSR stepsize, which greatly reduces the computer processing time.
} 
Similar to the ideas in Lutz and Bühlmann (2006), Blumensath and Davies (2008) this work adapts CG such that the iteratively constructed conjugate basis yields solutions of increasing cardinality. In case the algorithm can be terminated in less than $D$ steps a sparse solution is obtained. Adapting CG in this way results in an efficient implementation of OMP, which is a greedy based heuristic to solve (3) as pointed out in the previous section. Although the focus in this work is on over-determined linear systems, notice that OMP is usually applied in the under-determined case.

\subsection{Conjugate gradient method}

We now briefly review the CG algorithm, further details can be found in Nocedal and Wright (2006). Consider a linear system $A w=b$ with a symmetric positive definite matrix, such as in (5). Given a starting point $w^{(1)} \in \mathbb{R}^{D}$ one can solve the linear system by iteratively minimizing over a set of conjugate directions $p$ by setting

$$
w^{(k+1)}=w^{(k)}+\eta^{(k)} p^{(k)},
$$

where $k$ is the iteration number and $\eta^{(k)}$ the minimizer along the direction $w^{(k)}+\eta^{(k)} p^{(k)}$.

One of the remarkable properties of the conjugate gradient method is its ability to generate very cheaply the set of conjugate directions. This is achieved by incrementally constructing the conjugate basis set according to the highest remaining gradient of the optimization problem. Computation is performed in a clever memory-friendly way, by using the property that a conjugate vector $p^{(k+1)}$ can be computed based on the previous conjugate vector $p^{(k)}$ only. Therefore, one can write $p^{(k+1)}=-c^{(k+1)}+\xi^{(k+1)} p^{(k)}$, where $\xi^{(k+1)}$ is some appropriate scalar. The new search direction is determined by a linear combination of the steepest descent (negative gradient) direction and the previous search direction. An outline of CG is shown in Appendix A.

In case some conditions are met (Nocedal and Wright 2006), can approximate using a significantly smaller number of iterations than $D$. In case this property is preserved while using a sparse conjugate directions basis, a sparse (approximate) solution can be obtained.

\subsection{Modified CG using sparse conjugate directions}

As mentioned previously our goal is to formulate an algorithm which approximately solves the constrained minimization problem in (3). The idea is to adapt CG such that it can produce sparse solutions by using a set of sparse conjugate vectors. The algorithm goes as follows:

- We iteratively construct a conjugate basis consisting of basis vectors of incremental cardinality, starting with $w^{(1)}=0$.

- The algorithm performs a globally optimal optimization according to each conjugate direction.

- The sequence of conjugate basis vectors is designed such that we can terminate the procedure after a small number of iterations, leading to a sparse solution.

We call this algorithm Sparse Conjugate Directions Pursuit (SCDP).

Figure 3 displays the convergence plots for steepest descent, conjugate gradient and sparse conjugate gradient executed on a 2-dimensional quadratic objective function associated with a linear system $A w=b$. It is seen that the first direction of SCDP is sparse (only parameter $(w)_{1}$ is nonzero) and that similar to CG only 2 iterations are needed to converge to the global optimum. Steepest descent needs more iterations to converge as is seen by the dotted line in Fig. 3. 


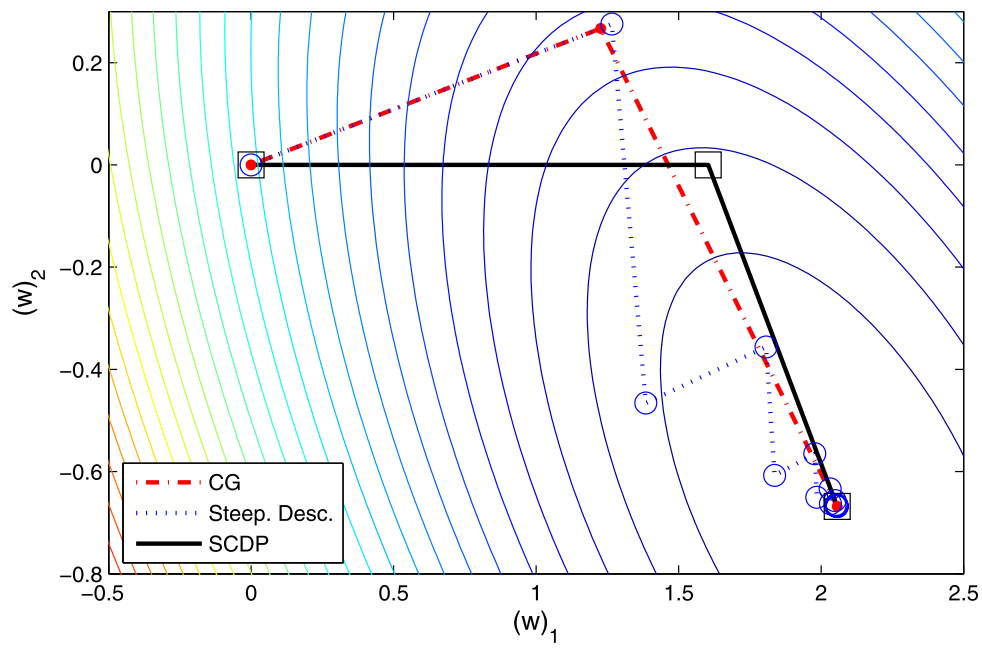

Fig. 3 Given the quadratic objective function associated with a linear system $A w=b$ of size $2 \times 2$, the solutions in different iterations of steepest descent (given an appropriate step size), conjugate gradient and sparse conjugate gradient are plotted. It is seen that the first direction of SCDP is sparse, only parameter $(w)_{1}$ is nonzero, and that similar to CG only 2 iterations are needed to converge to the global optimum. Steepest descent needs 9 iterations to converge

We now give a simple example indicating that SCDP for a specific problem outperforms LASSO. Given a matrix $X \in \mathbb{R}^{20 \times 15}$ and a vector $y \in \mathbb{R}^{20}$ both randomly sampled from a normal distribution with zero mean and unit standard deviation. Given a model size $l$ we try to minimize the following objective $\min _{w}\|X w-y\|_{2}^{2}$ such that $\|w\|=l$, hence aiming to be as close as possible to the Pareto front. The latter is created by performing an enumerative exhaustive search (which rapidly becomes impractical when $D$ increases) for finding the optimal subset of components given a cardinality. Hence, solving the problem defined in (3) exactly. This curve sets a lower bound on the Mean Squared Error (MSE), $\|X w-y\|_{2}^{2}$. Next SCDP, LASSO, and a de-biased LASSO are applied to $\left(X^{T} X+\varepsilon I\right) w=X^{T} y$ with $\varepsilon=10^{-9}$ to ensure numerical stability. Each curve is averaged over 100 randomizations. In Fig. 4 MSE values are plotted as a function of the cardinality for each of the methods. It is clearly observed that SCDP is closest to the Pareto front. The LASSO deviates more from the Pareto front since it introduces an undesirable bias due to adding an additional $L_{1}$ penalization term to the optimization objective. SCDP does not have such bias. For the LASSO it is therefore advised to use a second de-biasing step (Figueiredo et al. 2007) based on a least square estimate with the obtained sparsity pattern. As seen in Fig. 4 adding a de-bias step improves performance. Note that for (de-biased) LASSO, different values of $\lambda$ might give the same sparsity pattern. In Fig. 4 only that sparsity pattern providing the least MSE value is plotted.

Although the focus of this paper is on solving over-determined linear systems, we now consider a compressed sensing example (using the same setup as in Figueiredo et al. 2007) to indicating that SCDP can work in the under-determined case. Consider a signal $w \in \mathbb{R}^{4096}$ consisting of 160 spikes with amplitude \pm 1 drawn at the top in Fig. 5. The measurement matrix $X \in \mathbb{R}^{1024 \times 4096}$ is created by first filling it with independent samples of a standard Gaussian distribution and then orthonormalizing the rows. The observation vector $y$ is generated using $y=X w+n$, where $n$ is noise drawn from a Gaussian distribution with zero mean and a variance of $\sigma^{2}$. The middle of Fig. 5 presents the estimated signal $w^{S C D P}$ which 


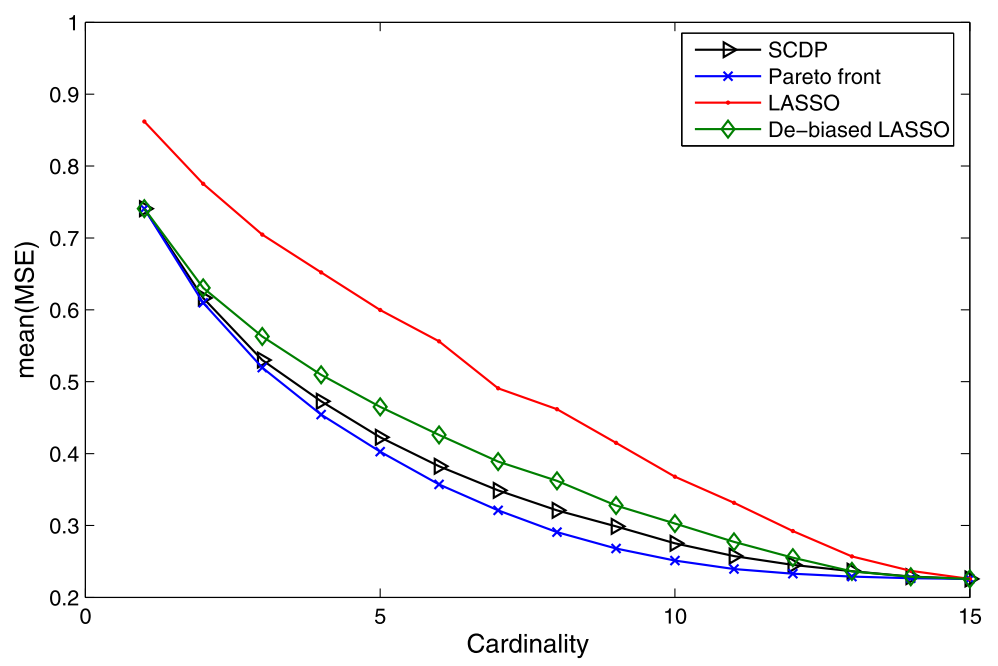

Fig. 4 Comparison of the optimal MSE achieved for a given cardinality of the solution, obtained by SCDP, the LASSO, de-biased LASSO and a Pareto front (the exact solution of the combinatorial problem (3)) is made based on a small randomly generated linear system of size $20 \times 15$. Results are averaged over 100 runs. The MSE of the linear system is plotted as a function of the number of nonzero components of $w$. Note that in case of the (de-biased) LASSO, multiple MSE values are available per model size. For visibility only the minimum values are plotted. In this example SCDP is closer to the Pareto front than its alternatives

is obtained using SCDP with a given measurement matrix $X$, and measurements $y$. Although $N$ the number of measurements is far less than $D$ the number of unknowns, SCDP successfully reconstructs the original sparse signal $w$. At the bottom of Fig. 5 the non-sparse least squares solution is given. Figure 6 presents the percentage of recovered signals for different variances. Per variance the results are averaged over 100 runs. In each run the initial filling of $X$ and the non-zero positions in $w$ are randomly chosen. A signal is recovered when the non-zero position (after appropriate thresholding) in the true signal $w$ match those in the estimated signal $w^{S C D P}$. The experiment is performed using both SCDP and LASSO. The regularization parameter $\lambda$ (used for LASSO) was set as suggested by Figueiredo et al. (2007). For small variances both methods are able to recover the original signal exactly. For larger variances a similar decrease in percentage of recovered signals is observed for both methods.

Algorithm 2 gives the pseudo-code of the basic version of SCDP (a more detailed version is described later). The outline of the algorithm resembles closely that of CG in Algorithm 5 in Appendix A, while an important difference lies in the construction of the conjugate basis vectors. Unfortunately, it is in general not possible to construct a new conjugate direction based on the previous solution only, as was the case for CG. As a consequence, the sparse conjugate directions pursuit algorithm has to store all previous directions.

We proceed by explaining how to exploit the sparse structure of the conjugate basis in order to efficiently compute $p^{(k+1)}$. Let $\pi:\{1, \ldots, D\} \rightarrow\{1, \ldots, D\}$ be a permutation which denotes the order in which the components of $A$ are selected in the algorithm. The next section will discuss how to select the components, but for now we assume a given $\pi$. Define a matrix $P \in \mathbb{R}^{D \times D}$ which might contain all search directions, or $P=\left(p^{(1)}, \ldots, p^{(D)}\right)^{T}$. The conjugate vectors are chosen such that the cardinality of each conjugate vector equals the iteration number. When we select $k$ columns of $P$ according to $\pi$ via selection matrix 

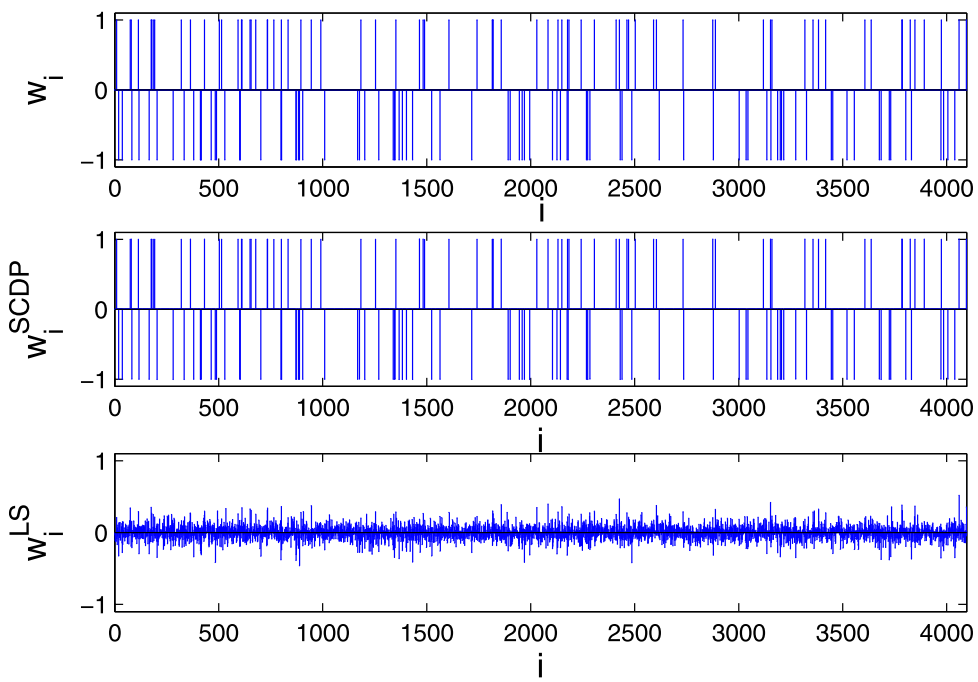

Fig. 5 Compressed sensing example. At the top the original signal sparse signal $w$ is plotted. The middle presents the estimated signal $w^{S C D P}$ which is obtained using SCDP with a given measurement matrix $X$, and measurements $y$. Although $N$ the number of measurements is far less than $D$, the number of unknowns, SCDP successfully reconstructs the original sparse signal $w$ given at the top. The bottom gives the least squares solution

Fig. 6 Compressed sensing example. For a set of variances for the Gaussian distributed noise which is added to $y$, a percentage of recovered signals is computed over 100 runs. Both LASSO and SCDP were ran

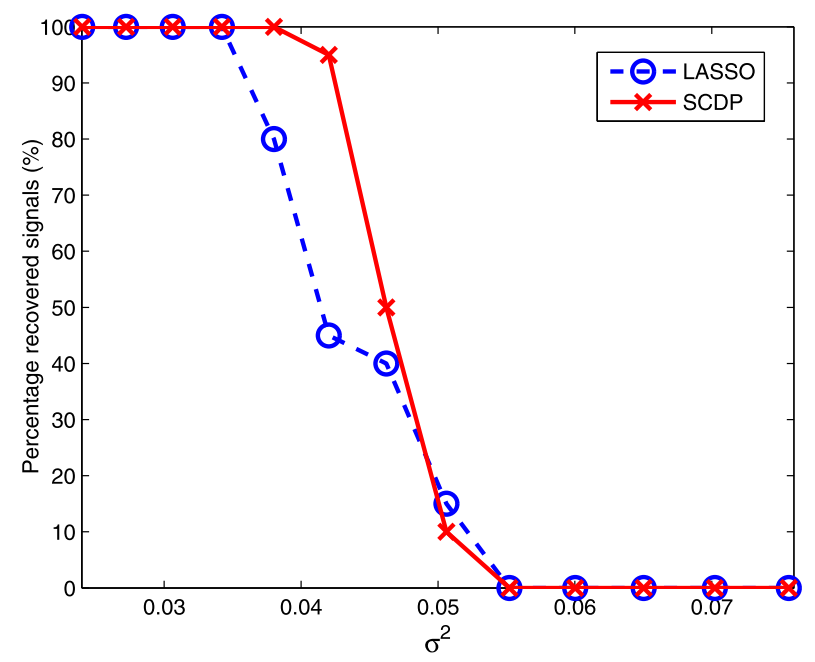

$\Sigma^{(k)} \in \mathbb{R}^{D \times k} ; \Sigma^{(k)}=\left(e_{\pi(1)} \ldots e_{\pi(k)}\right)$, the matrix structure is defined as follows

$$
P^{(k+1)}=\Sigma^{(k) T} P \Sigma^{(k+1)}=\left(\begin{array}{ccccc}
\left(p^{(1)}\right)_{\pi(1)} & 0 & & & 0 \\
\left(p^{(2)}\right)_{\pi(1)} & \left(p^{(2)}\right)_{\pi(2)} & 0 & & 0 \\
\vdots & \vdots & \ddots & \ddots & \vdots \\
\left(p^{(k)}\right)_{\pi(1)} & \cdots & & \left(p^{(k)}\right)_{\pi(k)} & 0
\end{array}\right) .
$$




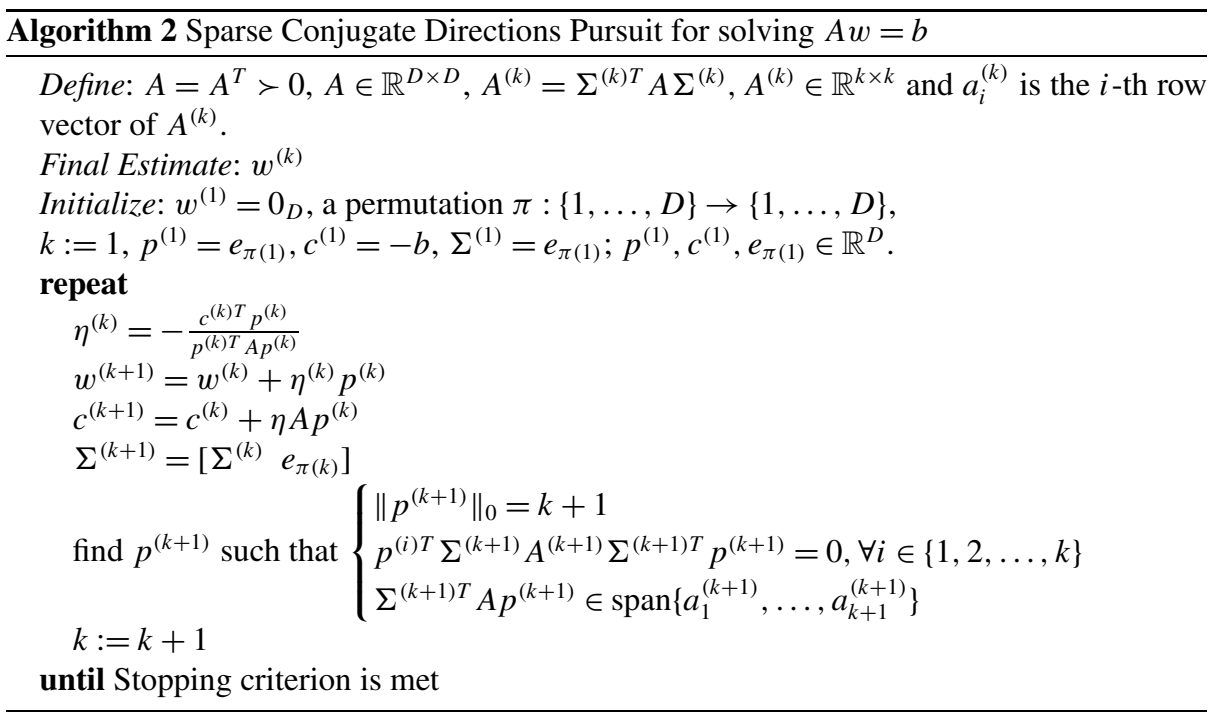

Now suppose the $k$ first conjugate vectors of $P$ are available then a vector $p^{(k+1)}$ is conjugate to this set with respect to $A$ when the following equation is satisfied

$$
\Sigma^{(k) T} P A p^{(k+1)}=0 .
$$

Since the set of vectors in $P$ possesses a certain sparsity pattern, (10) can be written as $P^{(k+1)} A^{(k+1)}\left(\Sigma^{(k+1) T} p^{(k+1)}\right)=0$ where $A^{(k+1)}=\Sigma^{(k+1) T} A \Sigma^{(k+1)}$. In order to obtain the desired sparse pattern for $p^{(k+1)}$, i.e. a cardinality that equals the iteration number, additional constraints are added. Hence, the new sparse conjugate direction $p^{(k+1)}$ must then satisfy the following

$$
\begin{aligned}
P^{(k+1)} A^{(k+1)} & \left(\Sigma^{(k+1) T} p^{(k+1)}\right)=0, \\
\text { such that } & \left\{\begin{array}{l}
\Sigma^{(k+1) T} A p^{(k+1)} \in \operatorname{span}\left\{a_{1}^{(k+1)}, \ldots, a_{k+1}^{(k+1)}\right\}, \quad \forall k, \\
\left\|p^{(k+1)}\right\|_{0}=k+1
\end{array}\right.
\end{aligned}
$$

where the span of a set of vectors in a vector space is the intersection of all subspaces containing that set, and $a_{i}^{(k+1)}$ is the $i$-th row or column vector of $A^{(k+1)}$. The first constraint determines which components of $p^{k+1}$ may be updated, i.e. those that correspond to the selected columns and rows in $A$. The second ensures that the cardinality of the solution equals the iteration number. As such, given a permutation $\pi$, one can compute a sequence of search directions by solving iteratively for $p^{(k+1)}$. Let us define $B^{(k+1)}=P^{(k+1)} A^{(k+1)}$, then 
Proposition 1 The matrix $B^{(k+1)}$ has ${ }^{7}$ an upper triangular matrix structure defined as follows

$$
B^{(k+1)}=\left(\begin{array}{ccccc}
b_{11}^{(k+1)} & b_{12}^{(k+1)} & \ldots & b_{1 k}^{(k+1)} & b_{1(k+1)}^{(k+1)} \\
0 & b_{22}^{(k+1)} & & & \vdots \\
\vdots & \ddots & \ddots & & \\
0 & \ldots & 0 & b_{k k}^{(k+1)} & b_{k(k+1)}^{(k+1)}
\end{array}\right)
$$

Proof Given a symmetric positive definite matrix $A$, we proof by induction over $k$ that the following equations hold

$$
\Sigma^{(k+1) T} p^{(i) T} a_{j}^{(k+1)}=0 \quad \text { for } i=2, \ldots, k+1 \text { and } \forall j=1, \ldots, i-1 .
$$

According to (10) for $k=1$ we have $p^{(1) T} A p^{(2)}=0$. Since $p^{(1)}$ contains only one nonzero entry this can be written as $z a_{1}^{(2) T} p^{(2)}=0$ were $z$ is an arbitrary constant or

$$
a_{1}^{(2) T} p^{(2)}=0 .
$$

In case $k=1$ equation $p^{(2) T} a_{1}^{(2)}=0$ holds due to (14). For $k=n$ we have

$$
\begin{cases}p^{(n+1) T} \Sigma^{(n+1)} a_{j}^{(n+1)}=0 & \text { for } \forall j=1, \ldots, n-1, \\ p^{(n) T} \Sigma^{(n+1) T} a_{j}^{(n+1)}=0 & \text { for } i=2, \ldots, n \text { and } \forall j=1, \ldots, i-1 .\end{cases}
$$

According to (10) the first set of equations hold in (15). Since $p^{(n)}$ has only $n$ nonzeros at entries corresponding with the first $n$ selected components of $A$, the following holds $\Sigma^{(n+1) T} p^{(n) T} a_{j}^{(n+1)}=\Sigma^{(n) T} p^{(n) T} a_{j}^{(n)}$. Hence using the induction hypothesis we have that the second set of equations in (15) are true. Since the equations in 3.2 hold, matrix $B^{(k+1)}$ has an upper triangular structure.

Remark that the first direction $p^{(1)}$ can be chosen arbitrarily (with the constraint that $\left.\left\|p^{(1)}\right\|_{0}=1\right)$ at the start of SCDP.

Given (10) the linear system (18) can be rewritten as

$$
B^{(k+1)}\left(\begin{array}{c}
\left(p^{(k+1)}\right)_{\pi(1)} \\
\vdots \\
\left(p^{(k+1)}\right)_{\pi(k+1)}
\end{array}\right)=0,
$$

where the first and second constraint in (11) are met. Since this is an under-determined system with $k+1$ parameters and $k$ equations we choose the parameter value $\left(p^{(k+1)}\right)_{\pi(k+1)}$ to be 1 in each iteration without loss of generality. If we define

$$
U^{(k+1)}=\left(\begin{array}{cccc}
b_{11}^{(k+1)} & b_{12}^{(k+1)} & \ldots & b_{1 k}^{(k+1)} \\
0 & b_{22}^{(k+1)} & & \vdots \\
\vdots & \ddots & & \\
0 & \ldots & 0 & b_{k k}^{(k+1)}
\end{array}\right),
$$

\footnotetext{
${ }^{7} b_{i j}$ denotes the $i j$-th element of matrix $B$.
} 
the following linear system can be written

$$
U^{(k+1)}\left(\begin{array}{c}
\left(p^{(k+1)}\right)_{\pi(1)} \\
\vdots \\
\left(p^{(k+1)}\right)_{\pi(k)}
\end{array}\right)=-\left(\begin{array}{c}
b_{1(k+1)}^{(k+1)} \\
\vdots \\
b_{k(k+1)}^{(k+1)}
\end{array}\right) .
$$

Because $U$ is an upper triangular matrix (Proposition 1) this linear system can be easily solved by backward substitution (Press et al. 1993).

Remark 2 Note that SCDP is always started using $w^{(1)}=0_{D}$. In this way the cardinality of $w^{(k)}$ equals $k$ for $k=1, \ldots, D$.

\subsection{On the choice of the next component}

A very important step in the algorithm is the determination of the next search direction in which the matrix $\Sigma^{(k)}$ selects the components of $A$. In relaxation techniques such as GaussSeidel (GS) (Young 2003) one iteratively sets one residual in each iteration to zero and hopes that this results in turning the new tentative solution closer to the correct solution. In order to ameliorate the convergence speed one can use an ordering scheme in which the individual residuals of $c^{(k)}=A w^{(k)}-b$ are set to zero according to Southwell iteration (Young 2003) which means that the components of the largest residuals are selected first.

In SCDP we use the same idea. In each iteration we add a component of $A$ which has the largest absolute residual

$$
s^{(k+1)}=\underset{i \notin \mathcal{A}}{\arg \max }\left|\left(c^{(k)}\right)_{i}\right|,
$$

where $\mathcal{A}$ is the set of previously selected indices. In each iteration the largest residual $c^{(k)}$ will be set to zero, which might result in a rapidly decreasing objective.

\subsection{Computational complexity}

In Algorithm 3 a detailed version of SCDP is shown. As with CG the most expensive operation is the matrix vector product $A p^{(k)}$. Because in SCDP we have that $\left\|p^{(k)}\right\|_{0}=k$, this product has complexity term $\mathcal{O}(k D)$. Unlike CG there is no simple update rule for determining a new conjugate vector. SCDP therefore needs to determine a new conjugate direction based on all previous ones. The most expensive operation regarding the conjugate basis is the computation of matrix $B^{(k+1)}$. Fortunately, it is not needed to recompute it in each iteration. At iteration $k+1$ we can incrementally update $B^{(k+1)}$ as follows

$$
B^{(k+1)}=\left(\begin{array}{cc}
B^{(k)} & P^{\prime(k) T} a_{s^{(k)}}^{(k)} \\
\left(0_{k-1}^{T} p^{(k) T} a_{s^{(k-1)}}^{(k)}\right) & p^{(k) T} a_{s^{(k)}}^{(k)}
\end{array}\right),
$$

where $P^{\prime}(k)$ equals $P^{(k)}$ without the last 2 columns and $0_{k}$ is a vector containing $k$ zeros. This means that $B^{(k+1)}$ can be updated iteratively at a cost of $\mathcal{O}\left(k^{2}\right)$. This gives an overall computational complexity of $\mathcal{O}\left(k D+k^{2}\right)$ per iteration $k$ compared to $\mathcal{O}\left(D^{2}\right)$ for standard CG. For large linear systems, SCDP will be much faster than CG in case only a small number of iterations are needed. In terms of memory usage of the algorithm (without incorporating the storage of $A$ ) we have that CG just needs to store the single previous direction, residuals and parameter values which gives $\mathcal{O}(3 D)$. SCDP needs to store the residuals $c^{(k)}$, the 
Table 1 Comparisons of SCDP and $\mathrm{CG}$ in terms memory requirement, training cost in iteration $k$ and the cardinality of the parameter vector $w$ (number of nonzero elements)

\begin{tabular}{lll}
\hline & SCDP & CG \\
\hline Memory & $\mathcal{O}\left(D+2 k+k^{2}\right)$ & $\mathcal{O}(3 D)$ \\
Computation & $\mathcal{O}\left(k D+k^{2}\right)$ & $\mathcal{O}\left(D^{2}\right)$ \\
Cardinality & $k$ & $D$ \\
\hline
\end{tabular}

nonzero elements of $p^{(k)}$ and $w^{(k)}$ and matrix $B^{(k)}$ which then gives $\mathcal{O}\left(D+2 k+k^{2}\right)$. In iteration $k$ of SCDP the cardinality of the parameter vector $w^{(k)}$ is $k$ compared to (most likely) $D$ in case of CG. The results are summarized in Table 1. Remark that the number of iterations necessary to converge typically is much smaller than the data set size. This can be observed in Sect. 5 in e.g. Table 3 where average model sizes (see column \#PV) obtained using SCDP (applied to kernel models) on a number of data sets are presented. Since for SCDP in iteration $k$ the model size equals $k$ it can be seen that the number of iterations to converge is usually much smaller than the number of examples.

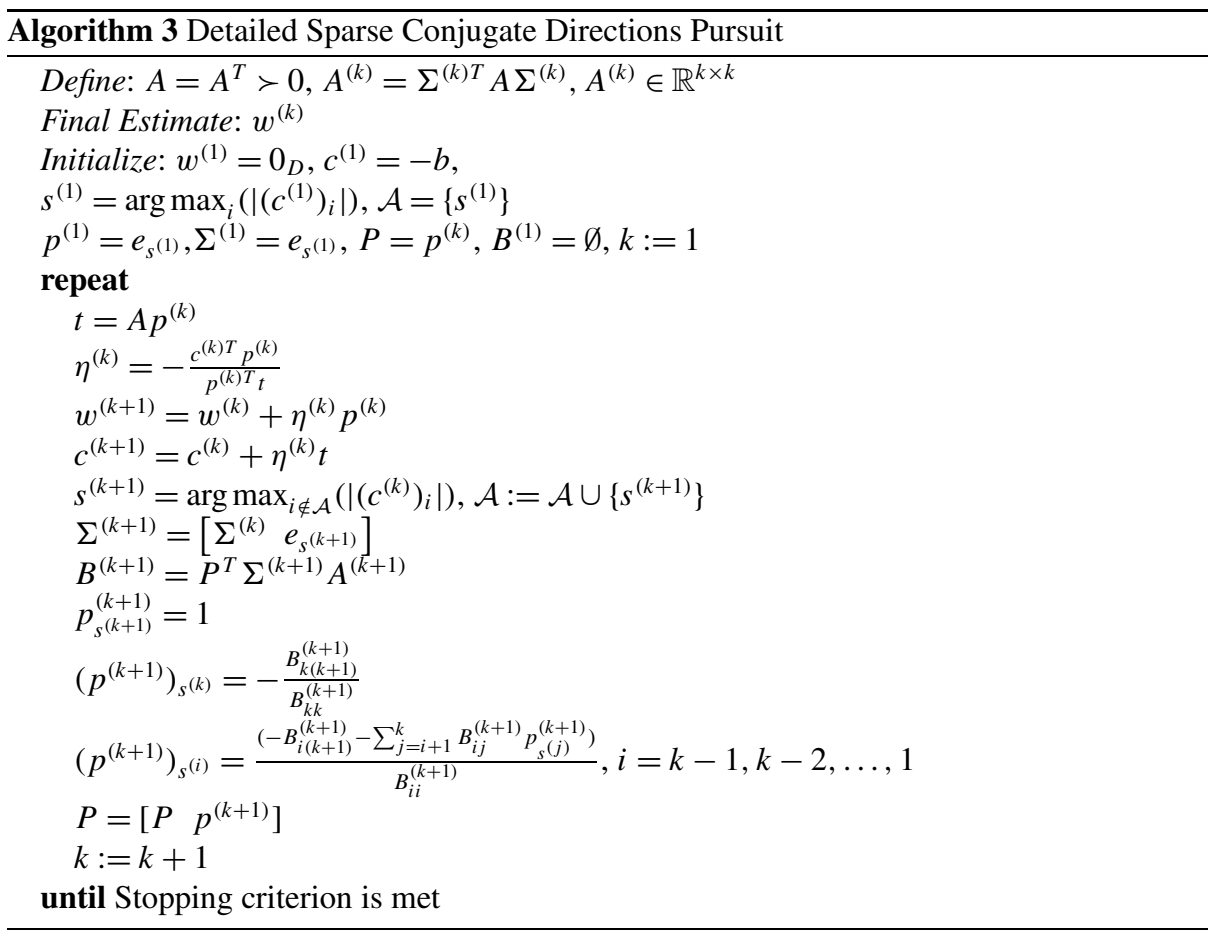

\subsection{Computational issues}

\subsubsection{Stopping criterion}

Defining a stopping criterion is to some extent dependent on the application. In the signal processing related literature algorithms are typically stopped when $\left\|r^{(k)}\right\|_{2}^{2}$ drops below some predefined threshold. In machine learning, model selection is usually performed using 
the error estimated on independent validation sets. Since the application in the next section resides in the latter context where the aim is to have a low misclassification rate on the training set and high generalization performance on unseen data, we shall rather choose the CV error to decide when to stop. In case the algorithm can be stopped early at iteration $k<D$ we have a model with a cardinality of $k$. In fact the CV as used to obtain the hyper-parameters is employed to determine the sparsity of the model as will be explained in Sect. 4.5.

\subsubsection{Probabilistic speed-up}

In order to decrease the computational complexity, algorithms similar to SCDP often only use a subsample of components to choose from. In case of large scale systems the main computational bottleneck of SCDP is to update $c^{(k)}$ (assuming that the algorithm can be stopped long before reaching $D$ ). In case $c^{(k)}$ is not used as a stopping criterion it is only used to add a new component to the active set $\left(s^{(k+1)}=\arg \max _{i \notin \mathcal{A}}\left(\left|\left(c^{(k)}\right)_{i}\right|\right)\right)$. Instead of using the full search space $\left(\left(c^{(k)}\right)_{i}, i=1, \ldots, D-k\right)$, for determining the novel nonsparse component in an iteration, of dimension $D-k$ only a random subset of size $\rho$ might be employed. This reduces the computation time. The resulting algorithm is called SCDP Probabilistic (SCDPP). In SCDPP there is no need to compute $A p^{(k)}$ entirely, but instead only $A^{(k)} \Sigma^{(k) T} p^{(k)}$ has to be computed and $c^{*(k)}=\Sigma^{* T} A \Sigma^{(k)} \Sigma^{(k) T} w^{(k)}-\Sigma^{* T} b$ where $\Sigma^{*} \in \mathbb{R}^{D \times \rho}$ is a randomly drawn selection matrix for the purpose of selecting $\rho$ components of $A$. Note that in case the probabilistic speed up is used, the update rule $c^{(k+1)}=c^{(k)}+\eta^{(k)} t$ (see Algorithm 3) cannot be used anymore since not all residuals are computed in each SCDP iteration.

In order to obtain a residual with a probability of 0.95 among the $5 \%$ largest residuals, we only need a random subset of size $\rho=59$ (Smola and Bartlett 2001; Popovici et al. 2005). This means that in each iteration we only compute 59 residuals and select from within that set the largest residual and corresponding component of $A$. In case the $A$ is available in memory, the training complexity can now approximately be written as $\mathcal{O}\left(\rho k+k^{2}\right)$. The total memory used by the algorithm is now $\mathcal{O}\left(\rho+2 k+k^{2}\right)$.

Note that repeating SCDP on a certain problem gives identical solutions. However, using the described speed-up affects reproducibility of the results since each run of the algorithm might give a different solution. As will be shown in the experimental section in some cases the results may deviate strongly from those of standard SCDP, resulting in less accurate predictions.

A more cleaver way of reducing computations might be to apply shrinking techniques such as used in the SVM literature (Joachims 1999). Leaving out examples (rows of $X$ ) with small corresponding $r^{(k)}$ (examples that are fitted correctly) in the computation of $c^{(k)}$ is not likely to harm the process of selection a new candidate. Therefor, it might be interesting to first select a set of examples with corresponding large $r^{(k)}$ values. Then do a number of iterations using lightweight updates only operating on the reduced set ( $\rho$ equals size of reduced set). Once in a while $r^{(k)}$ can be fully computed to change the reduced set.

\section{Application: using SCDP for sparse reduced LS-SVMs}

In this section a kernel-based learning method derived within the LS-SVM setting, is given. We will proceed as follows:

1. First the kernel-based LS-SVM primal-dual context is briefly reviewed.

2. Since this work aims at large-scale systems with $N \gg D$, it is more advantageous to solve in the primal space. 
3. In case of using kernels other than the linear one, the feature map in the primal formulation is not explicitly known. Therefore, an approximation is needed which in this work is obtained using the Nyström approximation (see Sect. 4.2).

4. As a result an over-determined linear system is obtained which may be solved using SCDP to get sparser models.

\subsection{Least squares support vector machines}

An overview of Least Squares Support Vector Machine (LS-SVM) models has been given in Suykens and Vandewalle (1999). Although the LS-SVM framework is studied in many other contexts as well, we will only consider it here in case of classification problems which is directly related to the regression case. Given a training set, the convex primal problem of the LS-SVM classifier can be formulated as

$$
\min _{w, e_{i}^{\prime}, b_{0}} \frac{1}{2} w^{T} w+\frac{\gamma}{2} \sum_{i=1}^{N} e_{i}^{\prime 2} \quad \text { such that } \quad y_{i} f\left(x_{i}\right)=1-e_{i}^{\prime}, i=1, \ldots, N,
$$

where a quadratic loss is used (instead of the hinge loss function typically employed in SVMs) where $f(x)=w^{T} \varphi(x)+b_{0}$. Another difference is the use of equality constraints instead of inequality constraints. The $e_{i}^{\prime}$ are slack variables allowing deviation form the target labels $y_{i}$. The formulation can be considered as a regression on the labels $y_{i}$ and is equivalent to

$$
\min _{w, e_{i}, b_{0}} \frac{1}{2} w^{T} w+\frac{\gamma}{2} \sum_{i=1}^{N} e_{i}^{2} \quad \text { such that } \quad f\left(x_{i}\right)=y_{i}-e_{i}, i=1, \ldots, N
$$

where $e_{i}^{\prime}=y_{i} e_{i}$. Given a positive definite kernel function $K: \mathbb{R}^{D} \times \mathbb{R}^{D} \rightarrow \mathbb{R}^{+}$with $K\left(x, x^{\prime}\right)=\varphi(x)^{T} \varphi\left(x^{\prime}\right)$ and a regularization constant $\gamma \in \mathbb{R}_{0}^{+}$, the solution to (22) is given by the following dual problem (according to Suykens et al. 2002b):

$$
\left(\begin{array}{c|c}
\Omega+\frac{1}{\gamma} I & 1_{N} \\
\hline 1_{N}^{T} & 0
\end{array}\right)\left(\begin{array}{c}
\alpha \\
\hline b_{0}
\end{array}\right)=\left(\begin{array}{c}
y \\
\hline 0
\end{array}\right),
$$

where $1_{N}=(1, \ldots, 1)^{T} \in \mathbb{R}^{N}, y=\left(y_{1}, \ldots, y_{N}\right)^{T}, \alpha=\left(\alpha_{1}, \ldots, \alpha_{N}\right)^{T}, \Omega_{i j}=K\left(x_{i}, x_{j}\right)$ and the identity matrix $I=\operatorname{diag}(1, \ldots, 1) \in \mathbb{R}^{N \times N}$. Note that this dual problem was also studied in Saunders et al. (1998) without the use of a bias term. Efficient CG based solvers exist to compute (23) (Chu et al. 2005).

\subsection{Fixed-size method: Nyström approximation and estimation in the primal}

Suppose one takes a finite dimensional feature map (e.g. a linear kernel $K\left(x, x^{\prime}\right)=x^{T} x^{\prime}$ ). Then one can equally well solve the primal (22) as the dual (23) problem. In fact solving the primal problem might be more advantageous for larger data sets where the dimension of the parameters $w \in \mathbb{R}^{D}$ is smaller compared to that of $\alpha \in \mathbb{R}^{N}$. In order to work in the primal space using a kernel function other than the linear one, it is required to compute an explicit approximation of the nonlinear mapping $\hat{\varphi}: \mathbb{R}^{D} \rightarrow \mathbb{R}^{N}$, such that $\Omega_{i j} \approx \hat{\varphi}\left(x_{i}\right)^{T} \hat{\varphi}\left(x_{j}\right)$. 
Based on Williams and Seeger (2001), the Nyström method is used to compute the approximated feature map $\hat{\varphi}_{i}: \mathbb{R}^{D} \rightarrow \mathbb{R}, i=1, \ldots, N$ for a training point, or for any new point $x^{*}$, with $\hat{\varphi}=\left(\hat{\varphi}_{1}, \ldots, \hat{\varphi}_{N}\right)^{T}$, is given as

$$
\hat{\varphi}_{i}\left(x^{*}\right)=\frac{1}{\sqrt{\lambda_{i}^{s}}} \sum_{j=1}^{N}\left(u_{i}\right)_{j} K\left(x_{j}, x^{*}\right),
$$

where $\lambda_{i}^{s}$ and $u_{i}$ are respectively the eigenvalues and eigenvectors of the kernel matrix $\Omega \in$ $\mathbb{R}^{N \times N}, \Omega_{i j}=K\left(x_{i}, x_{j}\right), x_{i}$ and $x_{j} \in \mathcal{X}_{\mathrm{TR}}$ with $\mathcal{X}_{\mathrm{TR}}$ the set of training examples.

Instead of using $\hat{\varphi} \in \mathbb{R}^{N}$, in Williams and Seeger (2001) it was motivated to use a subsample of size $M \ll N$ to compute an approximate feature map of size $M$. The $M$ vectors are called Prototype Vectors (PVs) which might, but do not necessarily, coincidence with training examples.

Let $M$ be the size of the PV set, $U=\left(u_{1}, \ldots, u_{M}\right)$ the square matrix of eigenvectors of $\Omega, u_{i}=\left(\left(u_{i}\right)_{1}, \ldots,\left(u_{i}\right)_{M}\right)^{T}$, and $\Lambda=\operatorname{diag}\left(\lambda_{1}^{s}, \ldots, \lambda_{N}^{s}\right)$ a diagonal matrix of nonnegative eigenvalues in decreasing order, $\Omega^{\prime} \in \mathbb{R}^{N \times M}, \Omega_{i j}^{\prime}=K\left(x_{i}, x_{j}\right), x_{i} \in \mathcal{X}_{\mathrm{TR}}, x_{j} \in \mathcal{X}_{\mathrm{PV}}$ with $\mathcal{X}_{\mathrm{PV}}$ the set of selected PVs. Then, the computation of the features in matrix notation can be written as

$$
\hat{\Phi}=\Omega^{\prime} U S
$$

with matrix $\hat{\Phi} \in \mathbb{R}^{N \times M}$ defined as

$$
\hat{\Phi}=\left(\begin{array}{ccc}
\hat{\varphi}_{1}\left(x_{1}\right) & \ldots & \hat{\varphi}_{M}\left(x_{1}\right) \\
\vdots & \ddots & \vdots \\
\hat{\varphi}_{1}\left(x_{N}\right) & \ldots & \hat{\varphi}_{M}\left(x_{N}\right)
\end{array}\right)
$$

and $S=\Lambda^{-\frac{1}{2}}$

Given $M \ll N$, the goal is to find an approximate solution for the over-determined system $\left(\hat{\Phi} \mid 1_{N}\right)\left(w^{T} \mid b\right)^{T}=y$. Solving (22) with an approximate feature map $\hat{\Phi}$ (i.e. the FS-LSSVM training) boils down to solving the following linear system (De Brabanter et al. 2010),

$$
\left(\begin{array}{c|c}
\hat{\Phi}^{T} \hat{\Phi}+\frac{1}{\gamma} I & \hat{\Phi}^{T} 1_{N} \\
\hline 1_{N}^{T} \hat{\Phi} & 1_{N}^{T} 1_{N}
\end{array}\right)\left(\begin{array}{c}
w \\
\hline b_{0}
\end{array}\right)=\left(\begin{array}{c}
\hat{\Phi}^{T} y \\
\hline 1_{N}^{T} y
\end{array}\right),
$$

where $\hat{\Phi} \in \mathbb{R}^{N \times M}$ is defined according to (26), $w \in \mathbb{R}^{M}$ are the model parameters and $y \in$ $\mathbb{R}^{N}$ the class labels in case of classification. Solving the primal as in (27) leads to a sparse representation.

Given an unseen data point $x^{*}$, the final classifier can be written as

$$
\hat{y}^{*}=\operatorname{sign}\left(w^{T} \hat{\varphi}\left(x^{*}\right)+b_{0}\right) .
$$

Given a value $M, \mathrm{PV}$ selection concerns the process of selecting a set of PVs which represent the training data distribution well. This set of PVs is then used to calculate the approximate feature map $\hat{\varphi}$. One approach might be to randomly select a subsample of the training set. Other techniques approach this selection using an appropriate selection criterion: (i) In Suykens et al. (2002b), De Brabanter et al. (2010) this is performed using a Rényi entropy based selection method; (ii) In Zhang et al. (2008) the observation is made that the 
Fig. 7 Given a (tuned) hyper-parameter set, a boxplot of the 10-fold CV misclassification score is plotted for the ripley data set using different sizes for $M$ (the number of PVs). The PVs are selected using $k$-center clustering. The boxes have lines at the lower quartile, median, and upper quartile values. The whiskers are lines extending from each end of the boxes to show the extent of the performances

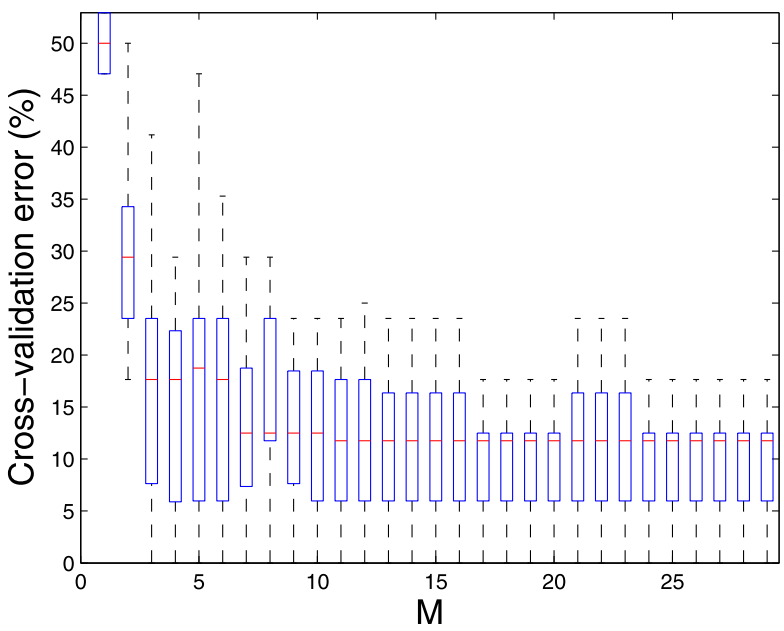

Nyström low-rank approximation depends crucially on the quantization error induced by encoding the sample set with landmark points. This suggests that one can simply use the clusters obtained with a $k$-center (such as $k$-means) algorithm, which finds a local minimum of the quantization error (note that using this technique the PVs do not necessarily coincidence with the training data). A simple and fast greedy algorithm which approximates the $k$-center clustering problem is proposed in Gonzalez (1985) and called farthest-point clustering.

According to Suykens et al. (2002b), De Brabanter et al. (2010) the CV prediction error of FS-LSSVM decreases with respect to the number of selected Prototype Vectors (PV) (or put in other words, cardinality of the model) until it does not change much anymore. In Suykens et al. (2002b), De Brabanter et al. (2010) empirical evidences indicates that this point of "saturation" might be obtained for values $M \ll N$. In Fig. 7 this is illustrated for the ripley data set (see Appendix B for more details about this data set). It is seen that the 10-fold cross-validation misclassification score does not improve much when more than 11 PVs are added.

Remark 3 Note that also in classical Radial Basis Function (RBF) network learning, methods have been developed where one separates the training of the centers from the training of the output weights. In RBF networks the centers are often determined by means of clustering (Chen et al. 1991,1992) or using a vector quantization method eventually in combination with self-organizing maps (Kohonen 1990) to visualize the input space of the network.

\subsection{Sparse conjugate directions pursuit applied to fixed-size models}

In FS-LSSVM the PV selection is performed prior to the actual model training (as a preprocessing step). This has the advantage that given a certain value for $M$ a model can be obtained very quickly. However, in case PV selection is linked to the actual learning goal one might expect better results (e.g. sparser solutions).

In this work the PV selection process is partially performed as a preprocessing and partially controlled by the model training. Motivated by the fact that (a) according to e.g. Vincent and Bengio (2002), Keerthi et al. (2006), Cawley and Talbot (2002), De Brabanter et al. (2010), Chen et al. (2009), Suykens et al. (2002b) the number of prototype vectors (or in 
related algorithms, support vectors) can be much smaller than the total number of training examples (b) and our observations, when using FS-LSSVM, that at a certain value for $M$ $(M \ll N)$ increasing the cardinality of the model does not change the prediction error much (as was seen in Fig. 7), one might first select a set of PVs with $M$ selected according to a criterion. An example of such criterion might be: choose $M$ such that $\Omega^{\prime} \in \mathbb{R}^{N \times M}$ still fits in memory. Having selected $M$ PVs, the learning boils down to solving an over-determined linear system as given in (27). This linear system may be solved using SCDP which then greedily selects the PVs (and calculates the model parameters) and, if stopped early, results in a model cardinality smaller than $M$. This early stopping (or the definition of an appropriate stopping criterion) is explained in Sect. 4.5.

Since $\hat{\Phi}$ is computed based on an eigenvalue decomposition (EVD), $\hat{\Phi}$ need to be recomputed in case an additional dimension (or an additional PV in this context) is added. This causes problems when implementing SCDP. In order to apply SCDP to (27) we first rearrange the FS-LSSVM system such that the parameters $w$ are solved up to a linear transformation resulting in the same predictions as the original formulation without the use of an EVD. This transformation does not require the computation of the approximate features $\hat{\Phi}$ explicitly. Therefore, it can be straightforwardly solved using the previously explained SCDP algorithm. This transformation is explained in Theorem 1.

Theorem 1 The linear system (27) is equivalent to

$$
\left(\begin{array}{c|c}
\Omega^{\prime T} \Omega^{\prime}+\frac{1}{\gamma} \Omega & \Omega^{\prime T} 1_{N} \\
\hline 1_{N}^{T} \Omega^{\prime} & 1_{N}^{T} 1_{N}
\end{array}\right)\left(\begin{array}{c}
\tilde{w} \\
\hline b_{0}
\end{array}\right)=\left(\begin{array}{c}
\Omega^{\prime T} y \\
\hline 1_{N}^{T} y
\end{array}\right),
$$

where $\Omega_{i j}=K\left(x_{i}, x_{j}\right)$ with $x_{i}, x_{j} \in \mathcal{X}_{\mathrm{PV}}$ and $\mathcal{X}_{\mathrm{PV}}$ the set of selected PVs, $\Omega \in \mathbb{R}^{M \times M} ; \Omega^{\prime} \in$ $\mathbb{R}^{N \times M}$ with $\Omega_{i j}^{\prime}=K\left(x_{i}, x_{j}\right), x_{i} \in \mathcal{X}_{\mathrm{tr}}$, and $x_{j} \in \mathcal{X}_{\mathrm{PV}}$ with $\mathcal{X}_{\mathrm{tr}}$ the set of training vectors; $\Omega U=U \Lambda ; \tilde{w}=U S w$ with $S=\Lambda^{-\frac{1}{2}}$. $\Lambda$ is assumed to be a diagonal matrix containing strictly positive eigenvalues.

Proof Recall that the computation of the approximate feature map $\hat{\varphi}$ is based on the eigenvectors and values of a reduced kernel matrix $\Omega U=U \Lambda, \Omega \in \mathbb{R}^{M \times M}$ resulting in

$$
\hat{\Phi}=\Omega^{\prime} U S
$$

where $\Omega^{\prime} \in \mathbb{R}^{N \times M}, \Omega_{i j}^{\prime}=K\left(x_{i}, x_{j}\right), x_{i} \in \mathcal{X}_{\text {tr }}, x_{j} \in \mathcal{X}_{\mathrm{PV}}$ with $\mathcal{X}_{\mathrm{PV}}$ the set of selected PVs and $\mathcal{X}_{\text {tr }}$ the set of training vectors and $S=\Lambda^{-\frac{1}{2}}$ (where we assume that the eigenvalues in $\Lambda$ are strictly positive). Combining (30) and (27) gives

$$
\begin{gathered}
\left(\begin{array}{c|c}
S U^{T} \Omega^{\prime T} \Omega^{\prime} U S+\frac{1}{\gamma} I & S U^{T} \Omega^{\prime T} 1_{N} \\
\hline 1_{N}^{T} \Omega^{\prime} U S & 1_{N}^{T} 1_{N}
\end{array}\right)\left(\frac{w}{b_{0}}\right)=\left(\begin{array}{c}
S U^{T} \Omega^{\prime} y \\
1_{N}^{T} y
\end{array}\right) \\
\Rightarrow\left(\begin{array}{c|c}
\Omega^{\prime T} \Omega^{\prime} U S+\frac{1}{\gamma}\left(S U^{T}\right)^{-1} & \Omega^{\prime T} 1_{N} \\
\hline 1_{N}^{T} \Omega^{\prime} U S & 1_{N}^{T} 1_{N}
\end{array}\right)\left(\frac{w}{b_{0}}\right)=\left(\begin{array}{c}
\Omega^{\prime} y \\
\hline 1_{N}^{T} y
\end{array}\right) \\
\Rightarrow\left(\begin{array}{c|c}
\Omega^{\prime T} \Omega^{\prime}+\frac{1}{\gamma} U S^{-2} U^{T} & \Omega^{\prime T} 1_{N} \\
\hline 1_{N}^{T} \Omega^{\prime} & 1_{N}^{T} 1_{N}
\end{array}\right)\left(\begin{array}{c}
\tilde{w} \\
b_{0}
\end{array}\right)=\left(\begin{array}{c}
\Omega^{\prime} y \\
1_{N}^{T} y
\end{array}\right),
\end{gathered}
$$


where

$$
\tilde{w}=U S w .
$$

Since $\Lambda=S^{-2}, \Omega U=U \Lambda$ and $U^{T} U=I$ we can write $U S^{-2} U^{T}=\Omega$. If we substitute this result in the latter linear system we get (29).

The classifier $\hat{y}^{*}=\operatorname{sign}\left(w^{T} \hat{\varphi}\left(x^{*}\right)+b_{0}\right)$ can be written as $\hat{y}^{*}=\operatorname{sign}\left(\Omega^{*} U S w+b_{0}\right)$ according to (30) where $\Omega^{*} \in \mathbb{R}^{1 \times M}, \Omega_{1 i}^{*}=K\left(x^{*}, x_{i}\right)$. Combining the latter and (32) we have $\hat{y}^{*}=\operatorname{sign}\left(\Omega^{*} \tilde{w}+b_{0}\right)$. This gives

$$
\begin{aligned}
\hat{y}^{*} & =\operatorname{sign}\left(w^{T} \hat{\varphi}\left(x^{*}\right)+b_{0}\right) \\
& =\operatorname{sign}\left(\sum_{i=1}^{M} \tilde{w}_{i} K\left(x^{*}, x_{i}\right)+b_{0}\right) .
\end{aligned}
$$

In order to ensure positive definiteness of the matrix in (29) we add a $L_{2}$ penalization term on the intercept term $b_{0}$ controlled by a small predefined positive regularization constant $v$ (in our experiments set to $10^{-8}$ ). Theorem 2 indicates the positive definite characteristic of the matrix in the considered linear system.

Theorem 2 For $v>0$ and $\gamma>0$, the matrix

$$
\left(\begin{array}{c|c}
\Omega^{\prime T} \Omega^{\prime}+\frac{1}{\gamma} \Omega & \Omega^{\prime T} 1_{N} \\
\hline 1_{N}^{T} \Omega^{\prime} & 1_{N}^{T} 1_{N}+v
\end{array}\right),
$$

is positive definite.

Proof An $N \times N$ real symmetric matrix $A$ is positive semi-definite if $p^{T} A p \geq 0$ for all nonzero vectors $p \in \mathbb{R}^{M}$ with real entries. By applying such multiplication to (2) we can write $^{8}$

$$
h=\left(d^{T} \mid c\right)\left(\begin{array}{c|c}
\Omega^{\prime T} \Omega^{\prime}+\frac{1}{\gamma} \Omega & \Omega^{\prime T} 1_{N} \\
\hline 1_{N}^{T} \Omega^{\prime} & 1_{N}^{T} 1_{N}+v
\end{array}\right)\left(\begin{array}{c}
d \\
- \\
c
\end{array}\right),
$$

which can be written as

$$
\begin{aligned}
h & =(1 c)^{T} 1 c+v c^{2}+2\left((1 c)^{T} \Omega^{\prime} d\right)+\left(\Omega^{\prime} d\right)^{T} \Omega^{\prime} d+\frac{1}{\gamma} d^{T} \Omega d \\
& =\left(1 c+\Omega^{\prime} d\right)^{T}\left(1 c+\Omega^{\prime} d\right)+\frac{1}{\gamma} d^{T} \Omega d+v c^{2} .
\end{aligned}
$$

Since the kernel matrix $\Omega$ by definition is positive semi-definite the product $d^{T} \Omega d$ is greater or equal than zero and hence because $\gamma>0, v>0$, we have $h>0$. Thus, the matrix (2) is positive definite.

\footnotetext{
${ }^{8}$ For clarity note that $\Omega^{\prime} \in \mathbb{R}^{N \times M}, \Omega \in \mathbb{R}^{M \times M}, 1 \in \mathbb{R}^{N}, c \in \mathbb{R}$, and $d \in \mathbb{R}^{M}$.
} 
Remark 4 In case the probabilistic speed-up is used (see Sect. 3.5.2), only a small fraction of the kernel matrix $\Omega^{\prime}$ need to be available. However, in each iteration kernel evaluations need to be recalculated. Keeping a cache of kernel evaluations to exclude or reduce the number of kernel recalculations will increase training time. Although intelligent caching methods (see the remark about shrinking in Sect. 3.5.2) could be defined, we simply choose an initial set of PVs, from which the SCDP algorithm can choose from, such that the corresponding entire $\Omega^{\prime}$ matrix fits in memory.

Remark 5 Note that our setup allows to select PVs other than those in the training set alone using e.g. $k$-center clustering.

\subsection{Model selection}

In order to assess the hyper-parameters such as the regularization constant $\gamma$ in (29) and the RBF-kernel bandwidth parameter $\sigma^{2}$, a $v$-fold CV based method is used. In the $v$-th iteration, a hyper-parameter pair is given a score according to some model selection criterion. A typical criterion expresses the number of misclassifications. However, we prefer a continuous function that is more amenable to numerical optimization routines. Therefore in this work the press statistic (Allan 1974) is used, to compute the $v$-fold CV error $f_{\mathrm{cv}}$, which is defined as

$$
f_{\mathrm{cv}}=\frac{1}{N_{v}} \sum_{v=1}^{N_{v}}\left(1_{N_{v}}^{T}\left(y\left(\mathcal{S}_{v}\right)-\hat{y}_{v}\left(\mathcal{S}_{v}\right)\right)^{2}\right),
$$

where the indices of the CV subsample sets $\left(N_{v}\right.$ the number of sets (folds)) are denoted as $\mathcal{S}_{v}, v=1, \ldots, N_{v}, \mathcal{S}_{1} \cap \cdots \cap \mathcal{S}_{N_{v}}=\emptyset$, and $\hat{y}_{v}=\left(\hat{f}_{v}\left(x_{1}\right), \ldots, \hat{f}_{v}\left(x_{N}\right)\right)^{T}$ with $\hat{f}_{v}$ the model which was trained in the $v$-th CV iteration. In Cawley (2006) the use of this press statistic for classification problems was empirically found to give good results.

To calculate a fast $v$-fold CV score, this work opts to use the simple approach given in De Brabanter et al. (2010) to calculate a fast $v$-fold CV (see De Brabanter et al. 2010 for references to other alternatives). The technique is described in Algorithm 4. Consider the shorthand notation $A_{\mathrm{f}} \tilde{w}=b_{\mathrm{f}}$ for the full linear system defined in (29). Consider a certain iteration in $\mathrm{CV}$ and let the matrix $A_{\text {tr }}$ be the matrix based on a subset of the training samples (all folds except one). Instead of computing $A_{\mathrm{tr}}^{(v)}$ (and corresponding $b_{\mathrm{tr}}^{(v)}$ ) from scratch in each $v$-th $\mathrm{CV}$ iteration, the following was proposed. At first the following results are computed: $\Omega^{\prime} \in \mathbb{R}^{N \times M}, \Omega \in \mathbb{R}^{M \times M}$ with $M$ the number of PVs (which do not have to coincidence with the training set), $A_{\mathrm{f}}$, and $b_{\mathrm{f}}$. Then based on the fact that we can use the same $\mathrm{PV}$ for each of the folds we can compute $A_{\mathrm{tr}}^{(v)}$ and $b_{\mathrm{tr}}^{(v)}$ for $\mathrm{CV}$ iteration $v$ as follows

$$
A_{\mathrm{tr}}^{(v)}=A_{\mathrm{f}}-\left(\begin{array}{c|c}
\Omega^{(v) T} \Omega^{\prime(v)} & \Omega^{\prime(v) T} 1_{N^{(v)}} \\
\hline 1_{N^{(v)}}^{T} \Omega^{\prime(v)} & N^{(v)}
\end{array}\right),
$$

with $N^{(v)}$ the number of examples of the $v$-th fold and $\Omega^{\prime(v)}$ is the selection of rows of $\Omega^{\prime}$ corresponding with the samples in the $v$-th fold. A similar approach is used for $b_{\mathrm{tr}}^{(v)}$

$$
b_{\mathrm{tr}}^{(v)}=b_{\mathrm{f}}-\left(\frac{\Omega^{\prime(v)} y^{(v)}}{1_{N^{(v)}}^{T} y^{(v)}}\right),
$$

where $y^{(v)}$ are the labels corresponding to the $v$-th fold. 
Remark 6 The matrices $\Omega^{\prime}$ and $\Omega$ for different kernel parameters can be computed more efficiently depending on which kernel function is used. For example consider the RBF kernel which is defined as $K\left(x, x^{\prime}\right)=\exp \left(n / \sigma^{2}\right)$ where $n=-\left\|x-x^{\prime}\right\|_{2}^{2}$. Instead of recomputing each kernel function from scratch one can proceed as follows: (i) compute $n$ only once; (ii) compute RBF kernel evaluations using $n$ with different values for $\sigma^{2}$.

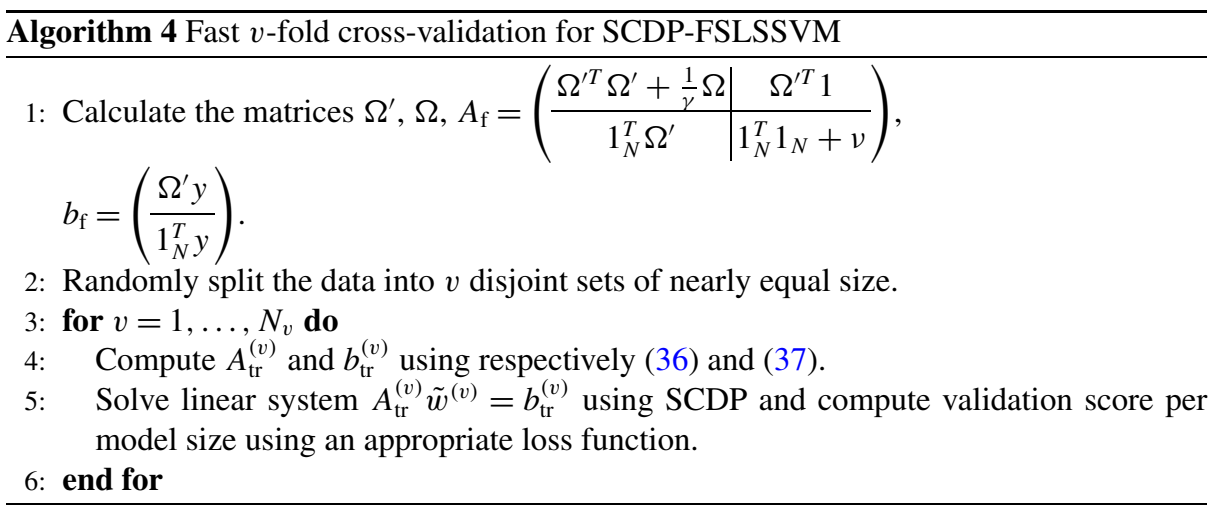

The actual hyper-parameter search process is a non-convex task which typically is tackled via a simple grid-search based procedure. The model selection score is evaluated at a set of points, forming a regular grid with even logarithmic spacing. An alternative strategy is using Nelder-Mead simplex optimization (Nelder and Mead 1965). This procedure can be used as long as the number of hyper-parameters is relatively small (in this work we tune two parameters, $\gamma$ and a kernel bandwidth $\sigma^{2}$ ). In order to determine good initial start values for this simplex method we use the method of Coupled Simulated Annealing with variance control (CSA) (Xavier de Souza et al. 2010) whose working principle was inspired by the effect of coupling in Coupled Local Minimizers (CLM) (Suykens et al. 2001) compared to the uncoupled case i.e. multi-start based methods. CSA is an extension to the well known Simulated Annealing (SA) algorithm (Rajasekaran 2000). The CSA method used in this paper is designed to easily escape from local optima and thus improves the quality of solution without compromising too much the speed of convergence. One of the largest differences with SA is that CSA features a new form of acceptance probabilities functions that can be applied to an ensemble of optimizers. This approach considers several current states which are coupled together by their energies in their acceptance function. Also, in contrast with classical SA techniques, parallelism is an inherent characteristic of this class of methods. In cases where a relative high number of hyper-parameters need to be tuned at once gradientbased methods are likely to be more efficient.

Remark 7 Note that to avoid the effects of the randomness in creating different $\mathrm{CV}$ partitions on the determination of the hyper-parameters, a fixed $\mathrm{CV}$ partition is chosen to minimize the model selection criterion.

\subsection{Determining the final model cardinality}

Let us first remark that in case the initial set of PVs equals the full training set, our method will approach the LS-SVM solution when the final model size is large. The same is true for 
the standard FS-LSSVM formulation. However, we are interested in having the cardinality of the final model as small as possible.

Since in iteration $k$ of the SCDP algorithm the number of nonzero model parameters is $k$, it is seen that the stopping criterion is directly related to the final model size. As mentioned earlier in machine learning, the aim is to have a model with a low misclassification rate on the training set and good generalization performance on unseen data. We therefore advocate the use of a validation error for deciding when to stop. For this purpose we can use the cross-validation performance as discussed in previous section. To save computer resources we keep track of the validation score as a function of $k$ (cardinality) and stop in case this function does not change much anymore. Hence, in case stopped early $(k<M+1$, with $M$ the size of the set of PVs) the learning problem is never solved using all PVs.

We proceed as follows. First an appropriate value for $k_{m}$, is chosen according to an appropriate criterion (e.g. maximum model size to be able to do real-time processing). For a given choice of hyper-parameters, the SCDP-FSLSSVM method is then applied on each separate $\mathrm{CV}$ fold using the following stopping criterion: run the algorithm until the number of iterations reaches $k_{m}$ or the validation score remains nearly the same. Combining the results for each fold gives an estimate of the $v$-fold $\mathrm{CV}$ error for each model size ranging from 1 to at most $k_{m}$. After combination of the results of the different folds, we select the final model size such that the $\mathrm{CV}$ criterion is the smallest value within one tenth of the standard deviation of the best cross-validation score (standard deviation was calculated from the different estimates computed using CV). In Hastie et al. (2001) (Sect. 7.10, page 216) a similar approach, referred to as the "one-standard-error", is given. This procedure can be repeated for different hyper-parameter values.

Remark 8 Note that in case the probabilistic speed-up is used, the random selection of candidate directions is fixed in order to exclude undesirable influence of the random effects in this tuning process. Thus, at iteration $k$, the different SCDP runs (using different values for the hyper-parameters) will choose the same random sets from which a new PV can be selected.

Remark 9 We found empirically that an adequate stopping rule is

$$
\frac{\left|\frac{1}{\Delta_{k}} \sum_{i=1}^{\Delta_{k}} f_{\mathrm{cv}}^{(k-i)}-f_{\mathrm{cv}}^{(k)}\right|}{\left|f_{\mathrm{cv}}^{(k)}\right|}<\epsilon,
$$

where $f_{\mathrm{cv}}^{(k)}$ defined as in (35) (where an extra superscript $(k)$ denotes the iteration dependency), and $\Delta_{k}$ is a fixed integer which determines the window size from which an average validation performance is calculated.

\subsection{Algorithmic parameters}

As illustrated in the previous section, four parameters: $M, \rho, \epsilon$ and $k_{m}$ control the processor and memory use of the algorithm and can have impact on the prediction accuracy of the final model. We therefore briefly summarize the effect of the different parameters in Table 2.

\subsection{Comparison of computational complexity}

Assuming an initial set of PVs of size $M$, the two most computational expensive operations in SCDP-FSLSSVM are: (i) the computation of $\Omega^{\prime T} \Omega$ in (29) with an approximate computational cost of $\mathcal{O}\left(N M^{2}\right)$ and (ii) solving the system using SCDP with an approximate 
Table 2 This table surveys the influence of the parameters $M, \rho, \epsilon$ and $k_{m}$ on the $\mathrm{SCDP}(\mathrm{P})$ algorithm

\begin{tabular}{ll}
\hline Parameter & Influence \\
\hline$M$ & Controls the initial size of the matrix in (29). We can set $M=N$ and select all training \\
data as initial PVs or can, according to some criterion, determine $M$ PVs which represent \\
the training set. The proposed framework allows the incorporation of PVs which do not \\
coincidence with the training data. \\
The size of the random subset out of which the PVs are selected in the probabilistic speed- \\
up case (SCDPP). The smaller $\rho$ the less $c(k)$ need to be computed in each iteration. \\
However, this means that different solution vectors with certain cardinality are possible \\
for a given linear system while for SCDP always the same solution is found. The larger $\rho$ \\
is, the more the solution will be similar to that of SCDP. \\
Stopping criterion parameter as defined in (38). At a certain point the addition of extra \\
PVs to the model has almost no effect on the validation performance. In this case the \\
algorithm can be stopped early which saves computer resources. In case enough resources \\
are available, probably the safest option is to set this parameter to $-\infty$. Afterwards the \\
cross-validation scores will be used to select the best cardinality. \\
Sets a maximum on the model size and training time.
\end{tabular}

cost of $\mathcal{O}\left(\sum_{k=1}^{M}\left(k N+k^{2}\right)\right)$, or using $\mathcal{O}\left(\sum_{k=1}^{M}\left(k \rho+k^{2}\right)\right)$ operations in case the probabilistic speed up (with $\rho=59$ in our experiments).

Assuming a "good" fixed set of PVs of size $M$, the cost of computing a classical FSLSSVM model is dominated by three parts: (i) the computation of $\hat{\Phi}$ (which includes an eigenvalue decomposition of the reduced kernel matrix $\Omega$ ) with an associated cost of $\mathcal{O}\left(M^{3}+M^{2} N\right)$, (ii) the computation of $\hat{\Phi}^{T} \hat{\Phi}$ in (27) with a computational cost of $\mathcal{O}\left(N M^{2}\right)$ and (iii) solving the linear system using for instance $\mathrm{CG}$ (in case the intercept term is regularized) with a cost $\mathcal{O}\left(k_{1} M^{2}\right)$ ( $k_{1}$ the number of iterations to converge).

\subsection{Relation to other methods}

\subsubsection{Relation to CDBoost}

In context of boosting, the Conjugate Direction Boosting (CDBoost) algorithm is proposed in Lutz and Bühlmann (2006). Using a similar idea of sparse conjugate vectors the authors extended the $L_{2}$-boosting method (Bühlmann and Yu 2003) and focus on linear regression (but also mention a classification example in the experimental section). They also showed that the CDBoost algorithm can be improved in terms of prediction accuracy by only taking a small fraction of the optimal step (shrinkage) along the conjugate direction, similar to the idea in FStR. ${ }^{9}$ Since this results in a higher computational cost (and extra tuning parameter) we did not consider this in this work. They also indicated that their framework could be generalized to the use of any base learner. Our work is different in the following areas:

- Our work specializes the SCDP idea to the kernel-based FS-LSSVM framework. Compared to Lutz and Bühlmann (2006), this work approaches the machine learning problem

\footnotetext{
${ }^{9}$ Due to the small stepsizes, the corresponding subspaces of the matrix in the linear system are no longer solved in a least squares sense. Thus, it may happen that a component already included in the model is rechosen. In this case no new conjugate directions can be found since there are only $k$ conjugate directions in a $k$-dimensional subspace. In Lutz and Bühlmann (2006) the authors propose to restart the algorithm. The actual $\hat{w}^{(k-1)}$ is set as the starting $\hat{w}^{(1)}$, all information about previous directions is deleted and the algorithm is started again.
} 
from a kernel-based context, use a ridge penalty (i.e. $\|w\|_{2}^{2}$ ) in a $L_{2}$-based objective and incorporate a separate intercept $\left(b_{0}\right)$ term into the model.

- CDBoost is not suited for large scale data sets. This work studies extensions to handle large scale data.

- Opposed to CDBoost, SCDP-FSLSSVM is presented with an efficient and complete model selection framework with a clear stopping criterion to automatically select the hyper-parameters, and final model size.

\subsubsection{Relation to other kernel-based methods}

In the literature there are several kernel-based methods which employ a greedy approach such as in SCDP-FSLSSVM in order to increase the model complexity during the training process. Some examples, which also work in the context of a $L_{2}$ loss function, include

- In Cawley and Talbot (2002) the primal LS-SVM objective (as in (21)) is approximated using a sparse approximate kernel expansion where only a relatively small number $M<$ $N$ of vectors contribute

$$
w=\sum_{x_{i} \in \mathcal{X}_{\mathrm{pv}}} \beta_{i} \varphi\left(x_{i}\right) .
$$

Eliminating in (22) the error variables $e_{i}$ by substituting the constraint in the objective and combining with (22), the same linear system is obtained as in (29). However, our approach is based on the FS-LSSVM method which gives additional insight in how to initially select PVs. Consequently, the underlying algorithm is different. Note that in the context of SVMs the same sparse approximate kernel expansion is proposed in Keerthi et al. (2006) resulting in a scheme which iteratively solves (29).

- Instead of approximating the primal using a sparse model, in Jiao et al. (2007) the authors directly approximate the dual LS-SVM formulation (23) and obtain a sparse model. Although, such a setup does not require the additional computation of a (numerical unstable) kernel matrix product $\Omega^{\prime T} \Omega^{\prime}$, we conclude from preliminary experiments ${ }^{10}$ that it requires more iterations to converge, resulting in a less sparse model and it requires comparable or even longer training times than SCDP-FSLSSVM (note that the authors in Jiao et al. 2007 made the same observation when comparing to Kernel Matching Pursuit (KMP) (Vincent and Bengio 2002)). In Jiao et al. (2007) the authors further elaborate on the comparison of this technique, Kernel Matching Pursuit (KMP), and Sparse Greedy Gaussian Process (SGGP) (Smola and Bartlett 2001).

- The idea of KMP is mainly to select basis functions in a greedy manner for nonregularized kernel least-squares problems. The authors describe 3 versions of KMP of which the back-fitting procedure is very similar to SCDP-FSLSSVM. Besides the absence of a regularization term and an intercept term, the order selection criterion is also different to that of SCDP-FSLSSVM. In case of KMP this is $s=\arg \max _{i \notin \mathcal{A}}\left|(r)_{i}\right|, r=\Omega w-y$ while for SCDP-FSLSSVM this is $s=\arg \max _{i \notin \mathcal{A}}\left|(c)_{i}\right|, c=\Omega^{T}(\Omega w-y)$.

- In Chen et al. (2009) the authors propose a general kernel learning framework based on Orthogonal Least Squares (OLS) regression. Given an orthogonal decomposition of the full kernel matrix $\Omega \in \mathbb{R}^{N \times N}, \Omega=Q R$ where $Q$ is the matrix with orthonormal columns,

\footnotetext{
${ }^{10}$ One such experiment was performed on the image data set (see Appendix B) were $N=1,300$, and $D=$ 18. Given a tuned set of hyper-parameters, applying SCDP directly on the dual (23) gave a model size of 961 while applying it on (29) gave a model size of 321. Both methods had comparable accuracies.
} 
the authors proceed by writing $\Omega w=b$ as $Q R w=b$ or $Q g=b$ and solving for $g=R w$ instead of $w$ (which is possible since the space spanned by the columns of $\Omega$ is identical to those spanned by columns in $Q$ ). Using the fact that $Q^{T} Q=I$, an efficient computation of $g$ and Leave-One-Out (LOO) scores can be obtained. Opposed to back-fitting (used by SCDP), OLS uses a pre-fitting scheme. This has impact on the PV selection procedure which is explained as follows. A new component is selected based on the LOO scores of all combinations of the not yet selected components with the current selected components. However, this pre-fitting requires the projection of all not yet selected columns to the subspace spanned by the selected components which is computationally more demanding than that of SCDP and not suitable for large-scale data sets.

\section{Experiments}

All SCDP-FSLSSVM experiments in this section are carried out in MATLAB. We empirically tested some of the algorithmic parameters for the SCDP-FSLSSVM algorithm discussed in the previous paragraphs and compare to state-of-the-art classification methods. The hyper-parameter $v$ which controls the regularization for the intercept term alone is set to $10^{-8}$ for all experiments. The other hyper-parameters are tuned using 10 -fold crossvalidation. For each experiment tuning was performed using the optimization procedure described in Sect. 4.4. For each experiment data is standardized to have zero mean and unit standard deviation. Details about the publicly available data sets used in the experiments are given in Appendix B. The SCDP-FSLSSVM MATLAB software can be downloaded from http://www.esat.kuleuven.be/sista/lssvmlab/SCDP/SCDP_FSLSSVM.zip. The SVM experiments are performed using the LIBSVM toolbox (Chang and Lin 2001).

\subsection{Prototype vector selection}

In order to illustrate the location of the selected PVs using SCDP-FSLSSVM, an experiment on the ripley data set is carried out. The SCDP-FSLSSVM method is initialized using a set of potential PVs containing all training data points. The binary decision boundary of the model obtained using SCDP-FSLSSVM (with tuned hyper-parameters) on the two-class ripley data set is presented in Fig. 8. The RBF kernel was used. The circles display the selected prototype vectors. It is seen that these are distributed over all parts of the data. Hence, selecting an approximately uniformly distributed (over the training set) set of data points with size $M<N$ in order to speed-up the training process, might be a good choice.

\subsection{Small scale problems}

We first assess the performance of SCDP-FSLSSVM on thirteen public domain benchmark data sets as used in the study by Mika et al. (2000). The 100 random partitions of the data (20 in the case of the image and splice benchmarks) to form training and test sets as was used in that study, are also used here. Model selection is performed independently for each realization of the data set. The RBF kernel $\left(K\left(x, x^{\prime}\right)=\exp \left(-\left\|x-x^{\prime}\right\|_{2}^{2} / \sigma^{2}\right)\right)$ was used for all kernel methods. In Table 3 some misclassification rates and corresponding model cardinalities are shown. The column corresponding to SCDP-FSLSSVM reflects results when all training data points are used as initial PVs and no probabilistic speed-up is used. The column with acronym SCDP-FSLSSVM $\mathrm{PV}_{\mathrm{PV}}$ first selects $0.3 N$ PVs using the farthest-point clustering algorithm (Gonzalez 1985) (we call this $k$-center clustering) from which the final model 
Fig. 8 The figure presents the binary decision boundary of the model obtained using SCDP-FSLSSVM (with tuned hyper-parameters) on the two-class ripley data set. The RBF kernel was used. The circles display the selected prototype vectors

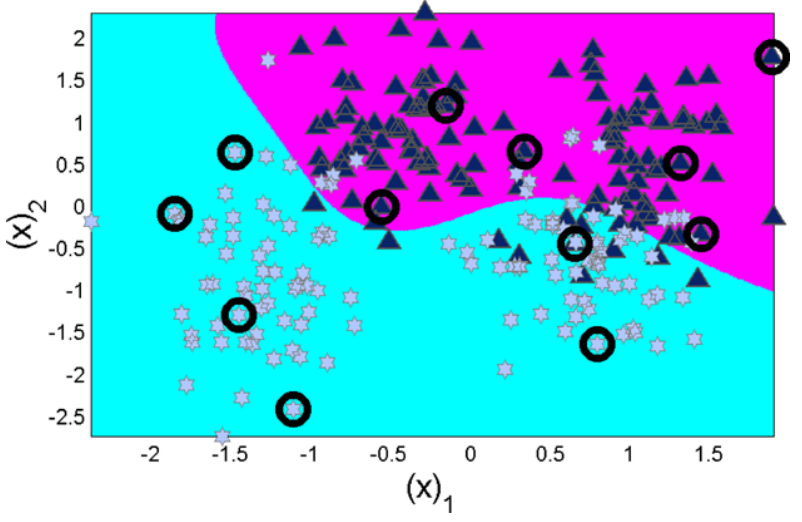

PVs are chosen, and no probabilistic speed-up was used. Note that Sect. 4.2 explains why $k$-center clustering is used for this purpose. In case of the third column, SCDPP-FSLSSVM training is performed using the probabilistic speed-up with a random subset of size $\rho=59$. The SCDP variants are compared to LS-SVM, SVM, and a sparse SVM (spSVM) (Keerthi et al. 2006) ${ }^{11}$ version. No preprocessing was done except standardization of the input samples to zero mean and unit variance. We endeavored making the framework for tuning the hyperparameter as much identical as possible. The same optimization procedures were used. In case of SVM and spSVM, misclassification rate (opposed to the press statistic) was used as the CV cost criterion. Both the SCDP variants and spSVM employed the same procedure to select the final model size (see Sect. 4.5).

Table 3 and Table 4 respectively give misclassification errors and computational cost (training time) for each data set. Note that making a direct comparison of running times is difficult here since SVM is implemented in C++ while the other alternatives are programmed in MATLAB. In LS-SVM the main computational load (i.e. solving a linear system) is handled using an optimized LAPACK source while for SCDP and spSVM the main computational load is in MATLAB. The test are performed using a computer with an Intel(R) Core(TM)2 Quad 2.66 GHz processor. To keep timing experiments as fair as possible all experiments were carried out single threaded.

It is seen that the sparse SCDP variants give comparable performance compared to the state-of-the-art methods: (non-sparse) LS-SVM, SVM and spSVM. When considering model sizes, all share comparable model complexities except for LS-SVM and SVM. By definition LS-SVM is non-sparse and SVM models have much larger cardinalities. Note that for SVM the model size indicates the number of nonzero dual variables while for the other alternatives the number of non-zero values in the primal model is meant. Notice that in case of SCDP-LSSVM $M_{\mathrm{PV}}$ in each run a different initialization of the k-center clustering algorithm is used. When comparing the misclassification scores to those of SCDP-FSLSSVM we can observe similar means and standard deviations indicating that the initial PV selection (selecting an initial PV pool having less than half the size of training set) including the initialization stage in $k$-center clustering has no significant effect on the misclassification rate.

Table 4 presents (averaged) training times in milliseconds. Since choice of hyperparameters affects training time and since the meaning of a "good" hyper-parameter set can

\footnotetext{
${ }^{11}$ From http://www.kyb.tuebingen.mpg.de/bs/people/chapelle/primal/.
} 
be different for each method, training times are measured using a tuned hyper-parameter set. From Table 4 it is seen that, while giving state-of-the-art accuracies, SCDP-FSLSSVM ${ }_{P V}$ in general is the fastest method. SCDP-FSLSSVM ${ }_{P V}$ decreases the training speed considerably by selecting an initial PV set of size $M=0.3 N$. Compared to the SCDP variants in most cases spSVM is much slower. Similar to the SCDP variants spSVM iteratively increases the model size starting with size 1 . A single iteration is more costly compared to the SCDP variants since a piecewise quadratic function needs to be solved using Newton's method. ${ }^{12}$ Remark that in case the subset size $(\rho)$ is close to the number of data points, the probabilistic speed-up (SCDPP-FSLSSVM) gives only a small or even negative gain compared to SCDPFSLSSVM. The advantage of using this probabilistic speed-up is seen more clearly in the experiments given in Sect. 5.3.

In case the probabilistic speed-up is used (SCDPP-FSLSSVM with $\rho=59$ ) the classification accuracies for data sets ringnorm and titanic deviate strongly from those of the other SCDP-FSLSSVM alternatives. The corresponding large standard deviations indicate that models with nontypical performance (outliers) are present. The same phenomena can be seen when plotting the Pareto fronts (see Fig. 9, and Fig. 10) for both SCDP- and SCDPPFSLSSVM given a single realization and tuned hyper-parameter set. SCDPP-FSLSSVM was repeated 50 times to display the boxplots. The boxes have horizontal lines at the lower quartile, median, and upper quartile values of the performances corresponding to the $50 \mathrm{ex}-$ periments. ${ }^{13}$ The dotted line is the SCDP-FSLSSVM Pareto front. It is seen that in most cases SCDPP-FSLSSVM performs slightly worse or similar in terms of misclassification rate compared to SCDP-FSLSSVM for different cardinalities. It is seen that for some data sets using SCDPP-FSLSSVM can give significant differences in terms of misclassification rate compared to the SCDP variant. In case $\rho$ is increased the SCDPP-FSLSSVM models converge towards those of SCDP-FSLSSVM but still, although less frequently, models with nontypical performance might occur. Note that for spSVM, which also uses a probabilistic speed-up $(\rho=59)$ this effect is only observed for the titanic data set. It is therefor adviced to use this probabilistic speed-up with care.

\subsection{Large scale problems}

The algorithm was additionally tested on two larger binary classification tasks. The results are shown in Table 5. The misclassification rates and corresponding model cardinalities are averaged over 5 random partitions of the data sets $(N / 3$ data points are used as test set and $2 N / 3$ examples as training set). No preprocessing was performed except for standardization of the input samples to zero mean and unit variance. Initially, 2,000 PVs were selected using $k$-center clustering. From Table 5 again it is seen that compared to SVM, SCDP-FSLSSVM and spSVM give similar performance while producing much sparser models.

These larger data sets are now used to illustrate the effect of the probabilistic speed-up on training time. In Fig. 11 for both the adult and gamma telescope data sets the misclassification rate is plotted in function of the training time for four SCDP-FSLSSVM variants. Notice that all methods more or less end-up in the same minimum. The basic SCDPFSLSSVM scheme lags behind its alternatives since the complete kernel matrix $\Omega$ needs

\footnotetext{
${ }^{12}$ As the authors suggested in Keerthi et al. (2006) the Newton optimization is only performed from time to time to save computer resources.

${ }^{13}$ Additionally the following markers are used in the boxplot. The whiskers are lines extending from each end of the boxes to show the extent of the rest of the data. Outliers ( +$)$ are data with values beyond the ends of the whiskers.
} 


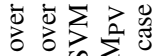

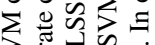

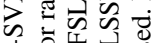

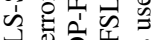

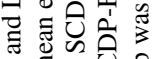

$\sum$ 흐요

至

की

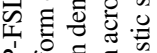

穴记

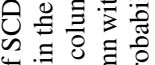

낭일

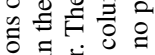

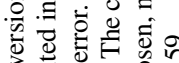

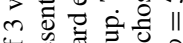

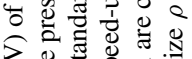

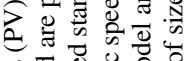

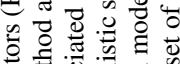

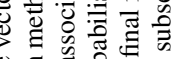

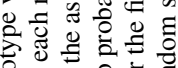

응 형영

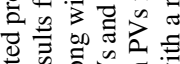

원 흘 등

送

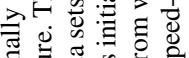

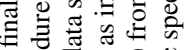

응 호을

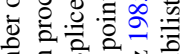

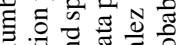

年

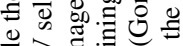

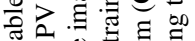

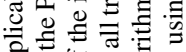

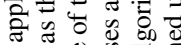

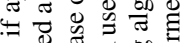

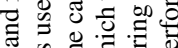

क人

这

흘

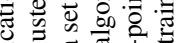

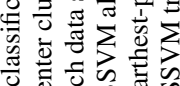

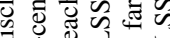

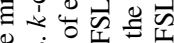

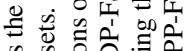

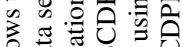

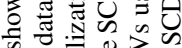

oi

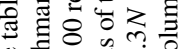

일

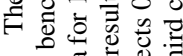

我

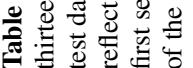

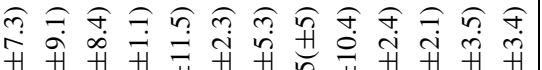

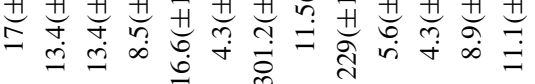

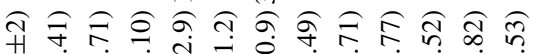

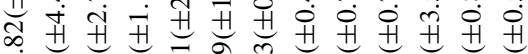

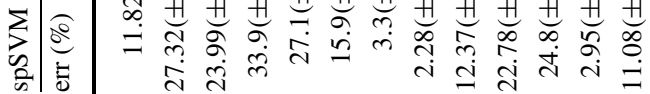

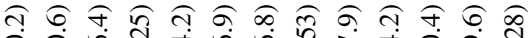

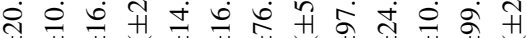

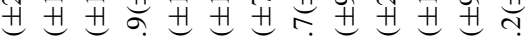

$\approx$ o

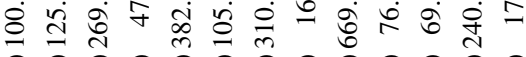

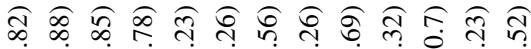

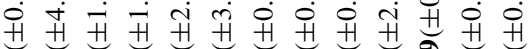

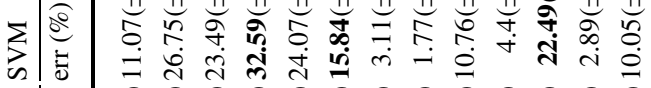

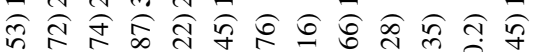

至

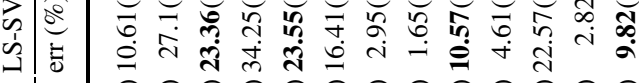

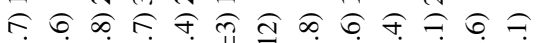

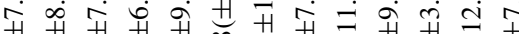

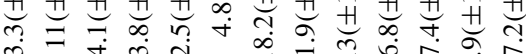

$a$

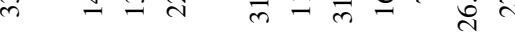

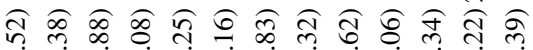

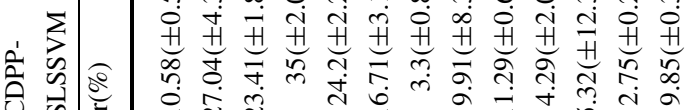

$\Leftrightarrow \dot{\pi} \pi$ ते

ิิ

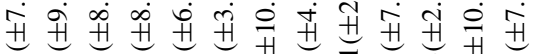

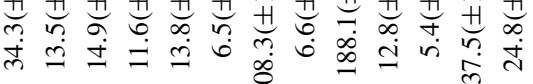

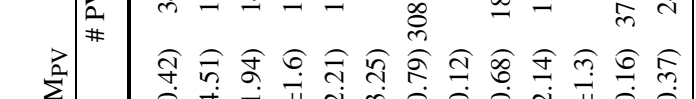

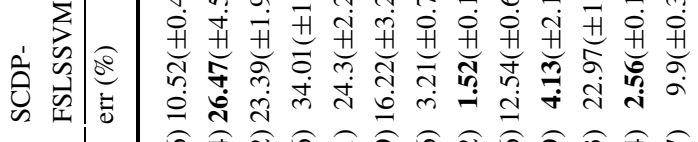

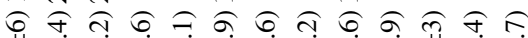

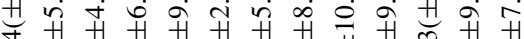

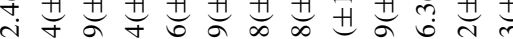

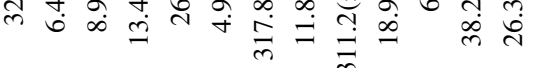

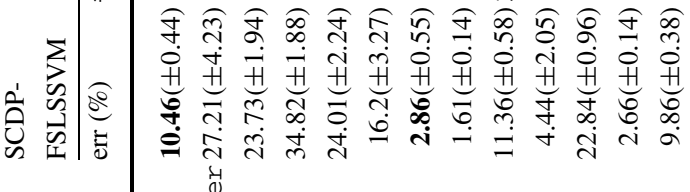

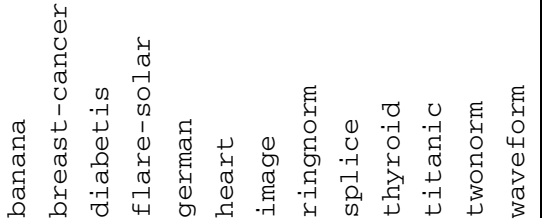




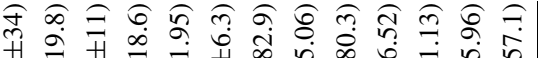

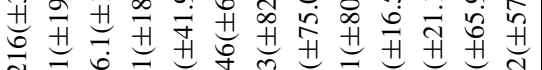

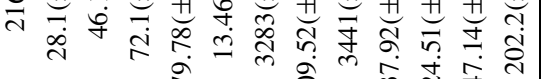

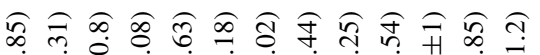

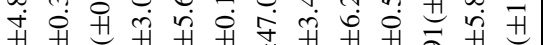

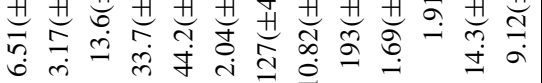

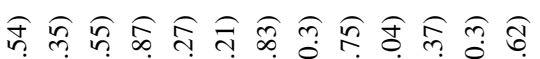

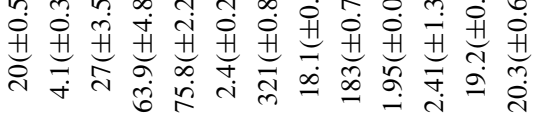
ธี

:

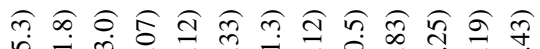
它

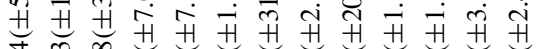

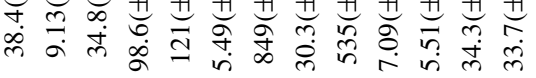

$\frac{n}{8}$

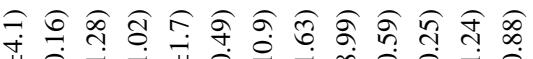

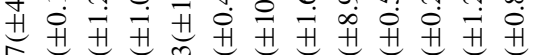

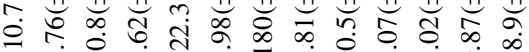
I.

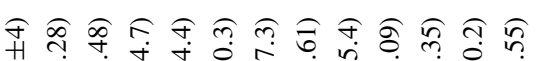

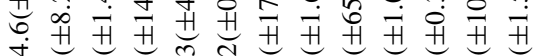

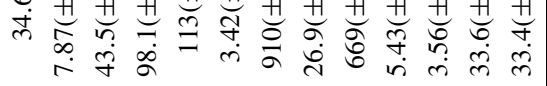




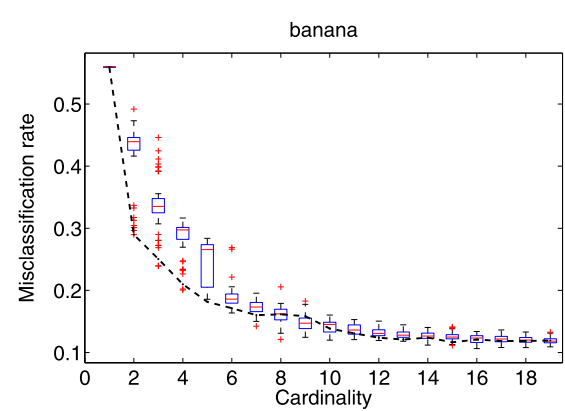

(a)

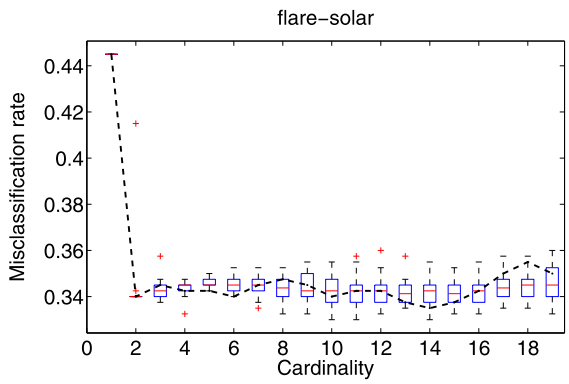

(c)

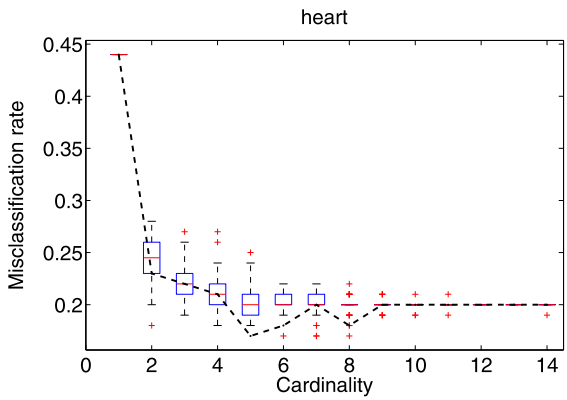

(e)

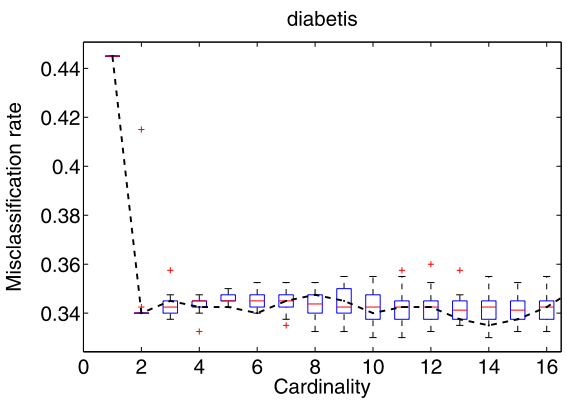

(b)

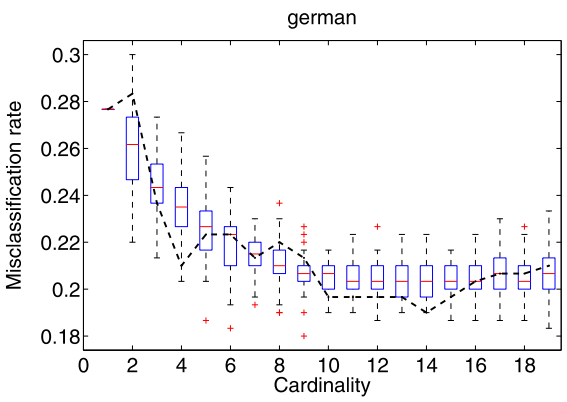

(d)

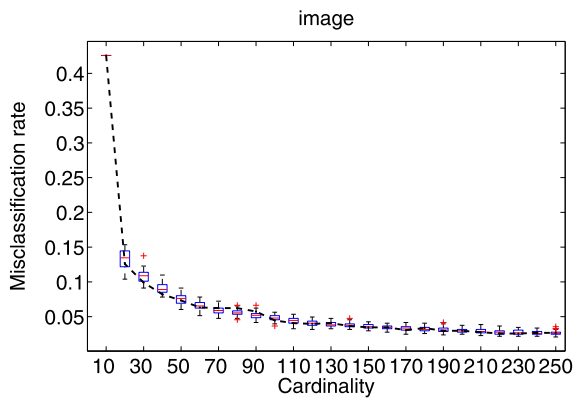

(f)

Fig. 9 Given a partition in CV and (tuned) hyper-parameter set, Pareto fronts are plotted for both SCDP- and SCDPP-FSLSSVM $(\rho=59)$ on data sets also used in Mika et al. (2000). SCDPP-FSLSSVM was repeated 50 times in order to make boxplots. The boxes have lines at the lower quartile, median, and upper quartile values of the performances corresponding to the 50 different experiments. The whiskers are lines extending from each end of the boxes to show the extent of the rest of the data. Outliers $(+)$ are data with values beyond the ends of the whiskers

to be computed in advance (since it is necessary in each iteration), before the SCDP part of the algorithm is started. The probabilistic speed-up in SCDPP-FSLSSVM does not require the latter. Therefor the SCDP algorithm can start iterating faster. As a consequence the misclassification rate decreases earlier. However, the course at which the misclassification rate is decreasing is not as steep as that of SCDP-FSLSSVM because of suboptimal PV 


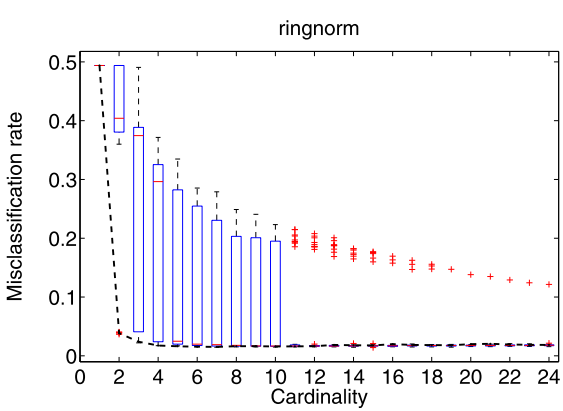

(a)

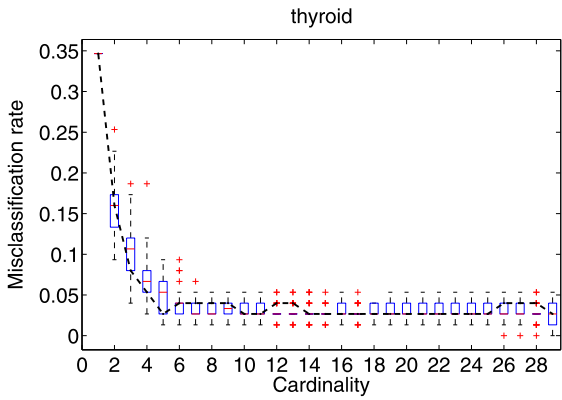

(c)

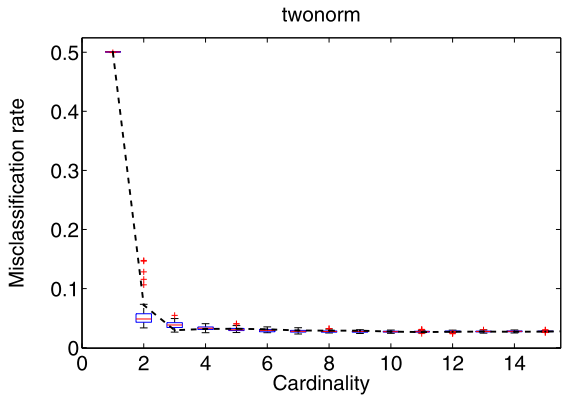

(e)

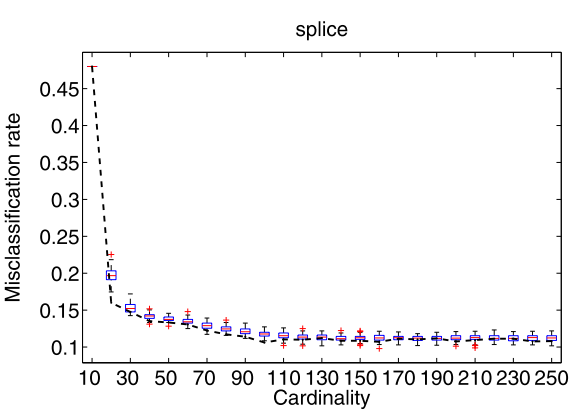

(b)

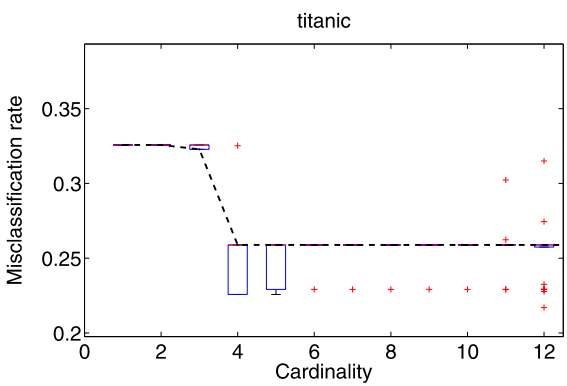

(d)

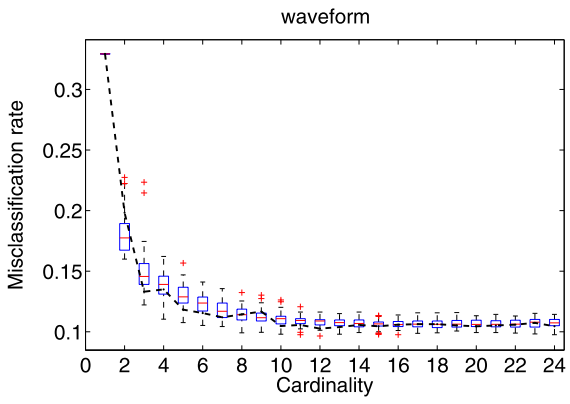

(f)

Fig. 10 Given a partition in CV and (tuned) hyper-parameter set, Pareto fronts are plotted for both SCDPand SCDPP-FSLSSVM $(\rho=59)$ on data sets also used in Mika et al. (2000). SCDPP-FSLSSVM was repeated 50 times in order to make boxplots. The boxes have lines at the lower quartile, median, and upper quartile values of the performances corresponding to the 50 different experiments. The whiskers are lines extending from each end of the boxes to show the extent of the rest of the data. Outliers $(+)$ are data with values beyond the ends of the whiskers

choices and more costly iterations. ${ }^{14}$ Since for SCDP-FSLSSVM $\mathrm{PV}_{\mathrm{PV}}$ only a reduced kernel matrix $\left(\Omega^{\prime}\right)$ needs to be precomputed, it starts faster with the SCDP part compared to SCDPFSLSSVM while having a similar course resulting in a faster conversion. When additionally

\footnotetext{
${ }^{14}$ Since for SCDPP-FSLSSVM no kernel matrix $(\Omega)$ is precomputed, small subsets of it need to be computed in each separate iteration. This makes a separate iteration more computationally involved. However, if the algorithm is terminated early at $k \ll N$ then possibly computing only a part of the kernel matrix $\Omega$ was sufficient. This might lead to important computational savings.
} 
Table 5 The misclassification rates, corresponding model cardinalities and training times (given a tuned hyper-parameter set) averaged over 5 random partitions of the data sets $(N / 3$ data points are used as test set and $2 N / 3$ examples as training set) for SCDP-FSLSSVM, spSVM as well as SVM are given. No preprocessing was performed except standardization of the input samples to zero mean and unit variance. Initially, 2, 000 PVs were selected using $k$-center clustering

\begin{tabular}{llrr}
\hline Method & & adult & \multicolumn{1}{l}{$\begin{array}{l}\text { gamma } \\
\text { telescope }\end{array}$} \\
& & & $13.9( \pm 0.27)$ \\
SCDP-FSLSSVMPV & \# PV & $166( \pm 41.6)$ & $778( \pm 58.9)$ \\
& $t_{\text {tr }}(\mathrm{s})$ & $10.7( \pm 2.6)$ & $44.4( \pm 1.6)$ \\
& err $(\%)$ & $14.53( \pm 0.14)$ & $13.3( \pm 0.12)$ \\
SVM & \# SV & $10,494( \pm 212)$ & $4,211( \pm 112)$ \\
& $t_{\text {tr }}(\mathrm{s})$ & $371.7( \pm 178.7)$ & $25.0( \pm 4.4)$ \\
& err $(\%)$ & $14.6( \pm 0.41)$ & $14.2( \pm 0.31)$ \\
spSVM & \# PV & $321( \pm 50.1)$ & $752( \pm 49.7)$ \\
& $t_{\text {tr }}(\mathrm{s})$ & $124.1( \pm 59.3)$ & $438( \pm 62.8)$ \\
\hline
\end{tabular}

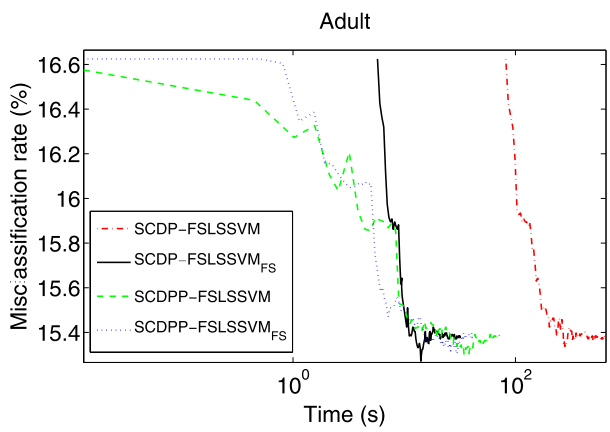

(a)

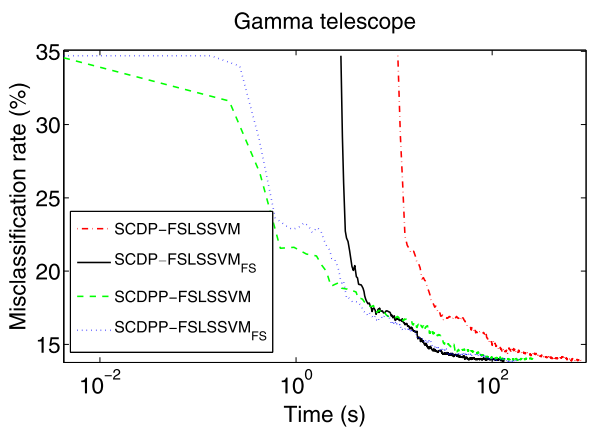

(b)

Fig. 11 Given a set of tuned hyper-parameters, time curves for SCDP-FSLSSVM, SCDPP-FSLSSVM, SCDP-FSLSSVMPV, SCDPP-FSLSSVMPV are given for the adult and gamma telescope data set

applying a probabilistic speed-up (SCDPP-FSLSSVM $\mathrm{PV}_{\mathrm{PV}}$ ) no faster conversion is observed.

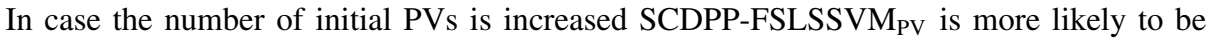
faster than SCDP-FSLSSVM PV $_{\text {. Compared to SCDPP-FSLSSVM, SCDPP-FSLSSVM }}$ is slightly more costly per iteration since 2 kernel matrices $\Omega^{\prime}$ and $\Omega$ need to get updated instead of one. However, choosing $\rho$ out of a reduced set, such as the case for SCDPPFSLSSVM $_{\mathrm{PV}}$, seems to pay off since faster convergence is observed compared to SCDPPFSLSSVM. Remark that the probabilistic speed-up versions need less memory compared to the other alternatives.

As mentioned in the first part, other methods exist to tackle the optimization problem (29) where a sparse model is desired. Examples include MP, LARS and LASSO. We have applied these methods for solving (29) and compared them to SCDP. Using LASSO, LARS and MP introduces a undesirable bias which is removed using an extra de-bias step. Hence, the coefficients of the nonsparse entries are recomputed using ordinary least squares. As such, we used the result of LASSO, LARS and MP only to select the PVs. This must be contrasted to SCDP where such de-bias step is not required. 


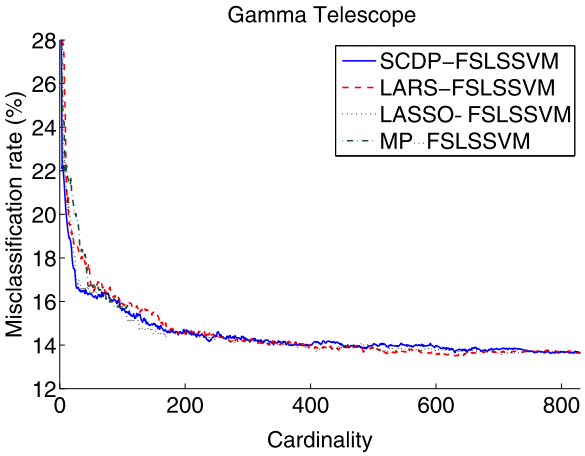

(a)

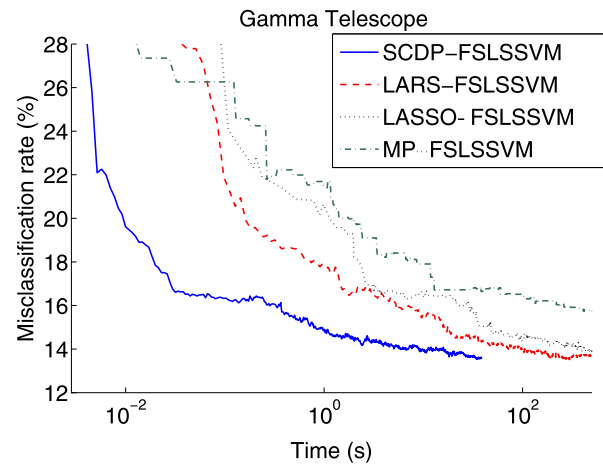

(b)

Fig. 12 Given a set of tuned hyper-parameters, Pareto fronts and time curves of SCDP, LARS, LASSO and MP applied to (29) are shown for the gamma telescope data set. The time curves show an average of 20 cumulative plots of the computation time for each of the alternatives per iteration

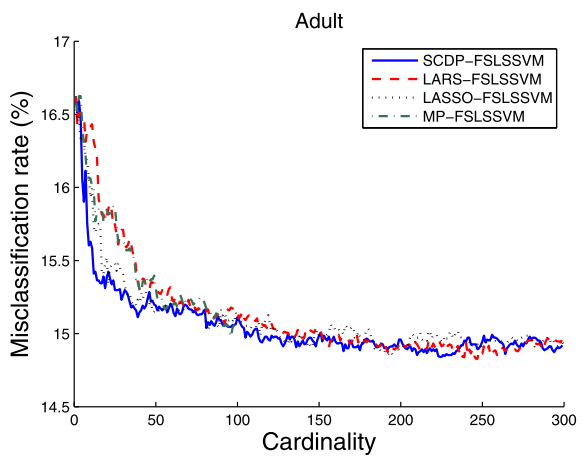

(a)

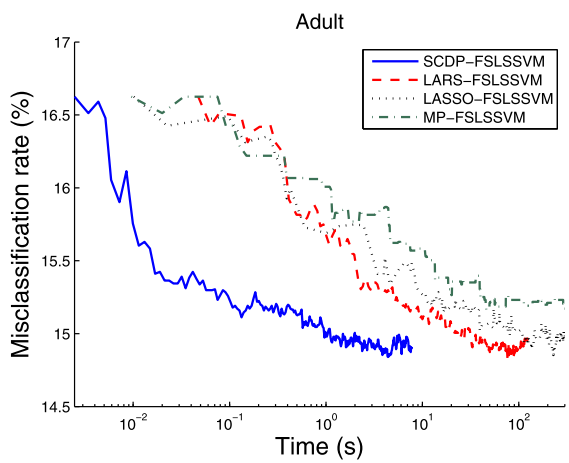

(b)

Fig. 13 Given a set of tuned hyper-parameters, Pareto fronts and time curves of SCDP, LARS, LASSO and MP applied to (29) are shown for the adult data set. The time curves show an average of 20 cumulative plots of the computation time for each of the alternatives per iteration

Given a set of (tuned) hyper-parameters, Pareto fronts are presented in Fig. 12(a) and Fig. 13(a) for respectively the gamma telescope and adult data sets. ${ }^{15}$ For both data sets the different Pareto fronts converge more or less to the same misclassification rate and similar model sparsity while training using SCDP is 7 to 10 times faster compared to the alternatives. This is mainly due to the fact SCDP does not require an additional de-bias step. As was expected, MP needs many more iterations to converge (convergence is not plotted) compared to its alternatives.

\footnotetext{
${ }^{15}$ Note that the LASSO in addition can also remove PV from the active set which means that for a certain cardinality multiple misclassification rates can be obtained. In order to enhance the clarity of the figures we only plotted the minimum misclassification rate per cardinality.
} 


\section{Conclusion}

In this manuscript we investigated a greedy heuristic, called Sparse Conjugate Directions Pursuit, for finding a sparse approximation to an over-determined linear system. We first discussed SCDP in relation to the literature of statistics, signal processing and machine learning. Subsequently, a detailed description of the SCDP implementation has been given and its computational complexity is discussed. Then it is explained how SCDP is applied to the LS-SVM framework, resulting in a sparse solution. A main advantage is that a range of model complexities (starting with a model that posses only one nonzero element in the weight vector) can be efficiently computed. We carefully examined SCDP's complexity in terms of memory and computational requirements and compared this to other sparse kernelbased methods. Finally, SCDP applied to FS-LSSVM was validated on a number of public available benchmark data sets. This results into the following main conclusions:

- Per iteration SCDP directly gives least-squares estimates on subspaces of $A$. This must be contrasted to other techniques such as LASSO which introduce an often undesirable bias due to a $L_{1}$ penalty. For such methods an extra de-biasing step might be employed to improve results.

- Misclassification errors are similar to those of LS-SVM while training times are much smaller and sparse models are obtained.

- Similar prediction accuracies as those of SVM were obtained, while SCDP usually produces much sparser models.

- SCDPP can improve upon the training speed but in case the size of the random subset was set to $\rho=59$, models with nontypical performance might occur.

- Compared to SVM and LS-SVM, SCDP-FSLSSVM is not a convex learning method. The solution of SCDP-FSLSSVM depends on the set of PVs selected by $k$-center clustering. However, for a given set of initial PVs (possibly all training data) and a zero solution vector $w^{(1)}=0_{D}$ in iteration 1, SCDP-FSLSSVM gives a unique solution.

- $k$-center clustering as a preprocessing step to select the initial set of PVs is a valuable tool to speed-up the training process. These PVs need not to be a member of the training set.

- We indicated that, when applying to FS-LSSVM, SCDP usually outperforms MP, LARS and LASSO in terms of speed while having a comparable accuracy.

Acknowledgements J.S. is a professor at the Katholieke Universiteit Leuven, Belgium. Research supported by Research Council KUL: GOA AMBioRICS, GOA MaNet, CoE EF/05/006 Optimization in Engineering (OPTEC), IOF-SCORES4CHEM, several PhD/post-doc \& fellow grants; Flemish Government: FWO: PhD/ postdoc grants, projects G.0452.04 (new quantum algorithms), G.0499.04 (Statistics), G.0211.05 (Nonlinear), G.0226.06 (cooperative systems and optimization), G.0321.06 (Tensors), G.0302.07 (SVM/Kernel), G.0320.08 (convex MPC), G.0558.08 (Robust MHE), G.0557.08 (Glycemia2), G.0588.09 (Brain-machine) research communities (ICCoS, ANMMM, MLDM); G.0377.09 (Mechatronics MPC), IWT: PhD Grants, McKnow-E, Eureka-Flite+, SBO LeCoPro, SBO Climaqs, POM, Belgian Federal Science Policy Office: IUAP P6/04 (DYSCO, Dynamical systems, control and optimization, 2007-2011); EU: ERNSI; FP7-HD-MPC (INFSO-ICT-223854), COST intelliCIS, EMBOCOM, Contract Research: AMINAL, Other: Helmholtz, viCERP, ACCM, Bauknecht, Hoerbiger.

\section{Appendix A: Conjugate gradient algorithm}

Since modifications to the standard conjugate gradient algorithm (Hestenes and Stiefel 1952) are proposed in this work, it is added in appendix. 


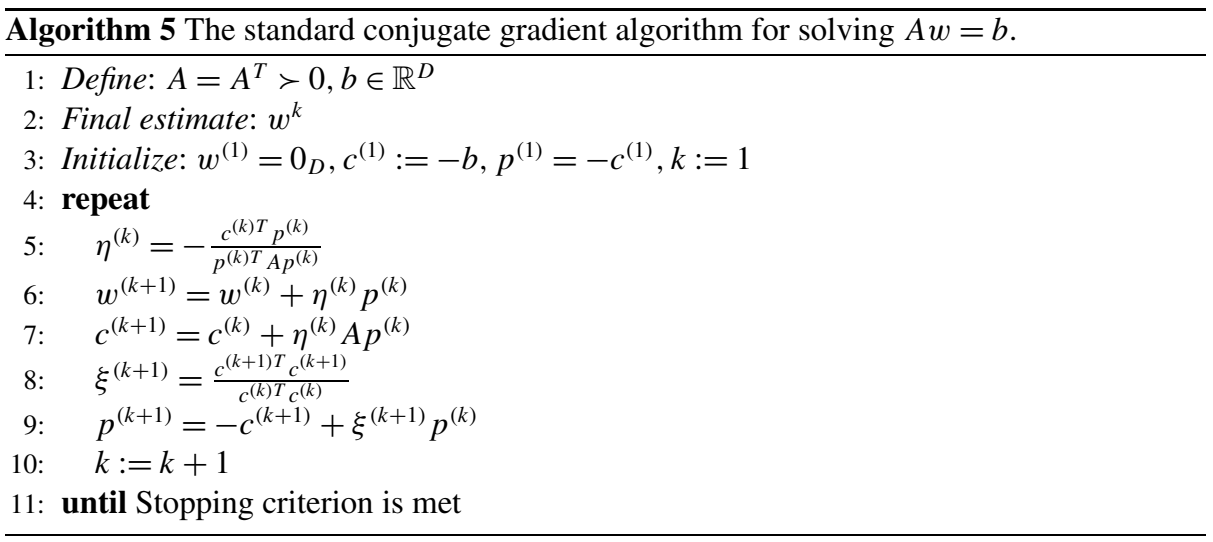

\section{Appendix B: Benchmark data sets}

This appendix describes the characteristics for each of the data sets used in this work. These are given in Table 6. The adult and gamma telescope data sets can be downloaded from http://kdd.ics.uci.edu, and the ripley data set can be found as the synthetic two-class problem at http://www.stats.ox.ac.uk/pub/PRNN/. Apart from those data sets, we also used modified versions of UCI data sets available from http://ida.first.fraunhofer.de/ raetsch. These data sets have a fixed number of partitions for each data set which makes comparison with other references a lot easier. Note that for adult, gamma telescope no fixed test set was given. During our experiments we chose to randomly divide all available data into a training and testing partition at a ratio of respectively $2 / 3$ and $1 / 3$ for several runs.

Table 6 Characteristics of the public domain data sets used in this work. Where $N_{\text {tr }}$ is the number of training data, $N_{\text {te }}$ the number of test data, $C$ the number of classes and $D$ the number of dimensions (features)

\begin{tabular}{lrrrr}
\hline data & \multicolumn{1}{c}{$N_{\text {tr }}$} & \multicolumn{1}{c}{$N_{\text {te }}$} & $C$ & $D$ \\
\hline adult & 30,148 & 15,074 & 2 & 14 \\
banana & 400 & 4,900 & 2 & 2 \\
breast-cancer & 200 & 77 & 2 & 9 \\
diabetis & 468 & 300 & 2 & 8 \\
flare-solar & 666 & 400 & 2 & 9 \\
gamma telescope & 12,680 & 6,340 & 2 & 10 \\
german & 700 & 300 & 2 & 20 \\
heart & 170 & 100 & 2 & 13 \\
image & 1,300 & 1,010 & 2 & 18 \\
ringnorm & 400 & 7,000 & 2 & 20 \\
ripley & 250 & 1,000 & 2 & 2 \\
splice & 1,000 & 2,175 & 2 & 60 \\
thyroid & 140 & 75 & 2 & 5 \\
titanic & 150 & 2,051 & 2 & 3 \\
twonorm & 400 & 7,000 & 2 & 20 \\
waveform & 400 & 4,600 & 2 & 21 \\
\hline & & & &
\end{tabular}




\section{References}

Allan, D. (1974). The relationship between variable selection and prediction. Technometrics, 16, $125-127$.

Blumensath, T., \& Davies, M. E. (2008). Gradient pursuits. IEEE Transactions on Signal Processing, 56(6), 2370-2382.

Bruckstein, A. M., Donoho, D. L., \& Elad, M. (2009). From sparse solutions of systems of equations to sparse modeling of signals and images. SIAM Review, 51(1), 34-81.

Bühlmann, P., \& Yu, B. (2003). Boosting with the 12-loss: Regression and classification. Journal of the American Statistical Association, 98, 324-339.

Candès, E. (2006). Compressive sampling. In Proc. of the international congress of mathematicians, Madrid, Spain.

Cawley, G. C. (2006). Leave-one-out cross-validation based model selection criteria for weighted LS-SVMs. In Proc. of the international joint conference on neural networks (IJCNN-2006), Vancouver, Canada (Vol. 2415, pp. 1661-1668).

Cawley, G. C., \& Talbot, N. L. C. (2002). A greedy training algorithm for sparse least-squares support vector machines. In Proc. of the international conference on artificial neural networks, Madrid, Spain (Vol. 2415, pp. 681-686).

Chang, C. C., \& Lin, C. J. (2001). Libsvm: a library for support vector machines. http://www.csie.ntu. edu.tw/cjlin/libsvm.

Chen, S., Billings, S. A., \& Luo, W. (1989). Orthogonal least squares methods and their application to nonlinear system identification. International Journal of Control, 50(5), 1873-1896.

Chen, S., Cowan, C., \& Grant, P. (1991). Orthogonal least squares learning algorithm for radial basis function networks. IEEE Transactions on Neural Networks, 2(2), 302-309.

Chen, S., Billings, S. A., \& Grant, M. (1992). Recursive hybrid algorithm for non-linear system identification using radial basis function networks. International Journal of Control, 55(5), 1051-1070.

Chen, S., Hong, X., Luk, B. L., \& Harris, C. J. (2009). Orthogonal-least-squares regression: A unified approach for data modelling. Neurocomputing, 72(10-12), 2670-2681.

Chen, S. S., Donoho, D. L., \& Saunders, M. A. (1999). Atomic decomposition by basis pursuit. SIAM Journal on Scientific Computing, 20(1), 33-61.

Chu, W., Keerthi, S. S., \& Ong, C. (2005). An improved conjugate gradient scheme to the solution of least squares SVM. IEEE Transactions on Neural Networks, 16(2), 498-501.

Daubechies, I., Vore, R. D., Fornasier, M., \& Gunturk, S. (2008). Iteratively re-weighted least squares minimization: Proof of faster than linear rate for sparse recovery. In Proc. of the information sciences and systems, Princeton, NJ (pp. 26-29).

De Brabanter, K., De Brabanter, J., Suykens, J. A. K., \& De Moor, B. (2010). Optimized fixed-size kernel models for large data sets. Computational Statistics \& Data Analysis, 54(6), 1484-1504.

de Kruif, B. J., \& de Vries, T. J. A. (2003). Pruning error minimization in least squares support vector machines. IEEE Transactions on Neural Networks, 14(3), 696-702.

Donoho, D. L. (2006). Compressed sensing. IEEE Transactions on Information Theory, 52(4), 1289-1306.

Donoho, D. L., \& Tsaig, Y. (2006). Fast solution of l1-norm minimization problems when the solution may be sparse (Tech. rep.). Stanford University.

Donoho, D. L., Tsaig, Y., Drori, I., \& Starck, J. (2006). Sparse solution of underdetermined linear equations by stagewise orthogonal matching pursuit (Tech. rep.). Stanford University.

Efron, B., Hastie, T., Johnstone, I., \& Tibshirani, R. (2004). Least angle regression (with discussion). Annals of Statistics, 32(2), 407-499.

Figueiredo, M. A. T., Nowak, R. D., \& Wright, S. J. (2007). Gradient projection for sparse reconstruction: Application to compressed sensing and other inverse problems. IEEE Journal of Selected Topics in Signal Processing, 1(4), 586-597.

Floyd, S., \& Warmuth, M. (1995). Sample compression, learnability, and the Vapnik-Chervonenkis dimension. Machine Learning Journal, 21, 269-304.

Gonzalez, T. (1985). Clustering to minimize the maximum intercluster distance. Theoretical Computer Science, 38, 293-306.

Hastie, T., Tibshirani, R., \& Friedman, J. (2001). The elements of statistical learning. Berlin: Springer.

Hestenes, M. R., \& Stiefel, E. (1952). Methods of conjugate gradients for solving linear systems. Journal of Research of the National Bureau of Standards, 49, 409-436.

Jiao, L., Bo, L., \& Wang, L. (2007). Fast sparse approximation for least squares support vector machine. IEEE Transactions on Neural Networks, 18(3), 685-697.

Joachims, T. (1999). Making large-scale SVM learning practical. In B. Schlkopf, C. Burges, \& A. Smola (Eds.), Advances in kernel methods-support vector learning. Cambridge: MIT Press, Chap. 11. URL http://www.cs.cornell.edu/People/tj/publications/joachims_99a.pdf. 
Keerthi, S. S., Chapelle, O., Decoste, D., Bennett, P., \& Parrado-hernández, E. (2006). Building support vector machines with reduced classifier complexity. Journal of Machine Learning Research, 7, 1493-1515.

Kim, S. J., Koh, K., Lustig, M., Boyd, S., \& Gorinevsky, D. (2007). An interior-point method for large-scale 11-regularized least squares. IEEE Journal on Selected Topics in Signal Processing, 1(4), 606-617.

Kohonen, T. (1990). The self-organizing map. Proceedings of the IEEE, 78(9), 1464-1480.

Lutz, R. W., \& Bühlmann, P. (2006). Conjugate direction boosting. Journal of Computational and Graphical Statistics, 15(2), 287-311.

Mallat, S. (1999). A wavelet tour of signal processing. New York: Academic Press.

Mika, S., Rätsch, G., Weston, J., Schölkopf, B., Smola, A., \& Müller, K. R. (2000). Invariant feature extraction and classification in feature spaces. Advances in Neural Information Processing Systems, 12, 526-532.

Moghaddam, B., Weiss, Y., \& Avidan, S. (2006). Spectral bounds for sparse PCA: exact and greedy algorithms. Advances in Neural Information Processing Systems, 18, 915-922.

Möller, M. F. (1993). A scaled conjugate gradient algorithm for fast supervised learning. Neural Networks, $6,525-533$.

Natarajan, B. K. (1995). Sparse approximate solutions to linear systems. SIAM Journal on Computing, 24, $227-234$

Nelder, J. A., \& Mead, R. (1965). A simplex method for function minimization. Computer Journal, 7, 308313.

Nocedal, J., \& Wright, S. J. (2006). Numerical optimization (2nd ed.). Berlin: Springer.

Osborne, M. R., Presnell, B., \& Turlach, B. A. (2000). A new approach to variable selection in least squares problems. IMA Journal of Numerical Analysis, 20, 389-403.

Pati, Y. C., Rezaifar, R., \& Krishnaprasad, P. S. (1993). Orthogonal matching pursuit: recursive function approximation with applications to wavelet decomposition. In Proc. of the 27-th annual Asilomar conference on signals, systems, and computers (pp. 40-44).

Popovici, V., Bengio, S., \& Thiran, J. P. (2005). Kernel matching pursuit for large datasets. Pattern Recognition, 38(12), 2385-2390.

Press, W. H., Cornell, B. P. F., Teukolsky, S. A., \& Vetterling, W. T. (1993). Numerical recipes in C: The art of scientific computing (2nd ed.). Cambridge: Harvard University Press.

Rajasekaran, S. (2000). On simulated annealing and nested annealing. Journal of Global Optimization, 16, 43-56.

Saunders, C., Gammerman, A., \& Vovk, V. (1998). Ridge regression learning algorithm in dual variables. In Proc. of the 15th int. conf. on machine learning (ICML-98) (pp. 515-521).

Smola, A. J., \& Bartlett, P. L. (2001). Sparse greedy Gaussian process regression. In Proc. neural information processing systems (Vol. 13, pp. 619-625).

Suykens, J. A. K., \& Vandewalle, J. (1999). Least squares support vector machine classifiers. Neural Processing Letters, 9(3), 293-300.

Suykens, J. A. K., Lukas, L., \& Vandewalle, J. (2000). Sparse approximation using least squares support vector machines. In IEEE Proc. int. symp. circuits syst., Geneva, Switzerland (pp. 757-760).

Suykens, J. A. K., Vandewalle, J., \& De Moor, B. (2001). Intelligence and cooperative search by coupled local minimizers. International Journal of Bifurcation and Chaos, 11(8), 2133-2144.

Suykens, J. A. K., De Brabanter, J., Lukas, L., \& Vandewalle, J. (2002a). Weighted least squares support vector machines: robustness and sparse approximation. Neurocomputing, 48(1-4), 85-105.

Suykens, J. A. K., Van Gestel, T., De Brabanter, J., De Moor, B., \& Vandewalle, J. (2002b). Least squares support vector machines. Singapore: World Scientific.

Tibshirani, R. (1996). Regression shrinkage and selection via the LASSO. Journal of the Royal Statistical Society. Series B, 58(1), 267-289.

Tropp, J. A. (2004). Greed is good: algorithmic results for sparse approximation. IEEE Transactions on Information Theory, 50(10), 2231-2242.

Vapnik, V. N. (1998). Statistical learning theory. New York: Wiley.

Vincent, P., \& Bengio, Y. (2002). Kernel matching pursuit. Machine Learning, 48, 165-187.

Williams, C. K. I., \& Seeger, M. (2001). Using the Nyström method to speed up kernel machines. Advances in Neural Information Processing Systems, 13, 682-688.

Xavier de Souza, S., Suykens, J. A. K., Vandewalle, J., \& Bollé, D. (2010). Coupled simulated annealing. IEEE Transactions on Systems, Man and Cybernetics. Part B, 40(2), 320-336.

Young, D. (2003). Iterative solution of large linear systems. New York: Courier Dover Publications.

Zeng, X. Y., \& Chen, X. W. (2005). Smo-based pruning methods for sparse least squares support vector machines. IEEE Transactions on Neural Networks, 16(6), 1541-1546.

Zhang, K., Tsang, I. W., \& Kwok, J. T. (2008). Improved Nyström low-rank approximation and error analysis. In Proc. of the 25th international conference on machine learning, Helsinki, Finland (pp. 1232-1239). 